\title{
Universal statistics of point vortex turbulence
}

\author{
By J. G. ESLER and T. L. A SHBEE \\ Department of Mathematics, University College London, \\ Gower Street, London WC1E 6BT, UK \\ j.g.esler@ucl.ac.uk
}

(Received 11 July 2015)

A new methodology, based on the central limit theorem, is applied to describe the statistical mechanics of two-dimensional point vortex motion in a bounded container $\mathcal{D}$, as the number of vortices $N$ tends to infinity. The key to the approach is the identification of the normal modes of the system with the eigenfunction solutions of the so-called hydrodynamic eigenvalue problem of the Laplacian in $\mathcal{D}$. The statistics of the projection of the vorticity distribution onto these eigenfunctions ('vorticity projections') are then investigated. The statistics are used first to obtain the density of states function and caloric curve for the system, generalising previous results to arbitrary (neutral) distributions of vortex circulations. Explicit expressions are then obtained for the microcanonical (i.e. fixed energy) probability density functions (pdfs) of the vorticity projections in a form that can be compared directly with direct numerical simulations of the dynamics. The energy spectra of the resulting flows are predicted analytically. Ensembles of simulations with $N=100$, in several conformal domains are used to make a comprehensive validation of the theory, with good agreement found across a broad range of energies.

The pdf of the leading vorticity projection is of particular interest because it has a unimodal distribution at low energy and a bimodal distribution at high energy. This behaviour is indicative of a phase transition, known as Onsager-Kraichnan condensation in the literature, between low energy states with no mean flow in the domain and high energy states with a coherent mean flow. The critical temperature for the phase transition, which depends on the shape but not the size of $\mathcal{D}$, and the associated critical energy are found. Finally the accuracy and the extent of the validity of the theory, at finite $N$, are explored using a Markov chain phase-space sampling method.

\section{Introduction}

Renewed interest in the classical problem of understanding the motion of point vortices has been stimulated by the results of recent experiments with quantum fluids 
(e.g. Neely et al. 2013). In the experiments, a Bose-Einstein condensate consisting of $O\left(10^{6}\right){ }^{87} \mathrm{Rb}$ atoms is confined by a trapping potential, and is excited by the application of a magnetic field. Vortices with quantized circulations are generated which, because three-dimensional motion is suppressed by the experimental geometry, subsequently evolve in two-dimensions. The canonical model for the evolution of the system is the Gross-Pitaevskii (de-focusing nonlinear Schrödinger) equation. However, in the experimental parameter regime, in which the healing length or core size of the vortices is much smaller than the trap scale, the point vortex model gives an excellent approximate description of the vortex motion (Bradley \& Anderson 2012). Related numerical work (Billam et al. 2014; Simula et al. 2014) has shown that the low wavenumber part of the incompressible energy spectrum can be accounted for using the point vortex system.

The methods of equilibrium statistical mechanics have been used to describe the statistics of point vortex motion ever since the pioneering study of Onsager (1949) (see also the review of Eyink \& Sreenivasan 2006). Most quantitative studies, following Joyce \& Montgomery (1973), have used mean field theory to obtain a predictive equation for the streamfunction $\psi$ of the time-mean flow induced by the vortices. The most well-known of these is the sinh-Poisson equation (see eqn. 3.26 below), which predicts the time-mean streamfunction $\psi$ of a uniform neutral vortex gas, consisting of equal numbers of positive and negative vortices with unit circulation.

A shortcoming of mean-field theory is that no information is provided about the fluctuations of quantities of interest about their mean. Additionally, as is easily discovered if numerical integrations of the point vortex equations are attempted, for randomly generated 'neutral gas' initial conditions, it is often the case that no time-mean flow emerges from the dynamics (i.e. $\psi=0$ ). Mean-field theory evidently reveals no useful information about these $\psi=0$ simulations. Additionally, sinh-Poisson solutions occur in positive / negative pairs, due to the $\psi \rightarrow-\psi$ symmetry of the equation. It can be inferred that the emergence of each pair of solutions, at low mean-field energies in the sinh-Poisson equation, can be associated with a spontaneous symmetry breaking of the no-flow $\psi=0$ solution. Understanding this symmetry breaking is a key focus of this work.

The spontaneous, symmetry breaking emergence of a mean circulation in the point vortex system is known as Onsager-Kraichnan condensation (e.g. Billam et al. 2014; Simula et al. 2014) in the physics literature. Condensation is widely observed in nonequilibrium systems that are related to the point vortex model, in the sense that they reduce to the point vortex model in a particular limit (e.g. the limit of small vortex size), with one clear example being the superfluids experiments described above. Additionally, however, condensation is observed in numerical simulations (e.g. Chertkov et al. 2007) and experiments (Paret \& Tabeling 1998; Shats et al. 2005) of both forced and unforced $2 \mathrm{D}$ turbulence in fluids, as well as in bounded magnetized plasmas (Bos et al. 2008), 
where its occurrence has been argued to be of importance for plasma confinement in tokomak experiments. One particularly interesting example of condensation occurs in numerical simulations of decaying two-dimensional Navier-Stokes turbulence in a square domain (Clercx et al. 1998, 2001). In this case condensation is associated with the fluid spontaneously and quite rapidly increasing its total angular momentum. By contrast, in a rectangular domain with sufficiently large aspect ratio, a dipole circulation with no angular momentum is observed to form instead.

Condensation can be viewed as the result of non-equilibrium processes such as repeated vortex merger (or annihilation in the case of superfluids), with the condensate emerging as the end state of the merger (or annihilation) process when a small number of vortices are left in the domain, in an equilibrium configuration. In fluid turbulence, however, Xiao et al. (2009) have shown that vortex merger alone cannot quantitatively account for the upscale transfer of energy entering the condensate structure. The work of Taylor et al. (2009), applying the earlier ideas of Chavanis \& Sommeria (1996), provides insight that condensation in fact has a statistical basis. They demonstrate that point vortex statistical mechanics can be used to predict the outcome of the Clercx et al. experiments, in particular explaining the transition between 'spin up' and dipole forming flows as the domain geometry is altered. In Taylor et al.'s theory, both the spin-up and dipole flows are examples of condensation, occurring when significant number of vortices remain present, with the spatial structure of the condensate in each case being the structure with maximum entropy according to the point vortex theory. A switch in the maximum entropy solution occurs when the aspect ratio of the rectangle exceeds $\approx 1.12$ thus accounting for the transition.

In order to understand fully the statistical basis of condensation, however, it is necessary to use an alternative approach to the mean-field theory used by Taylor et al. The cumulant expansion method of Pointin \& Lundgren (1976) is one method to obtain a richer statistical description of point vortex behaviour. Using cumulant expansion, depending on the scaling of the energy $E$ with the number of vortices as $N \rightarrow \infty$, either Joyce and Montgomery's mean-field theory is recovered (in the 'hydrodynamic' scaling limit, in which $e=E / N^{2} \Gamma_{0}^{2}$ is held constant as $N \rightarrow \infty$, where $\Gamma_{0}$ is the root-mean-squared vortex circulation), or a theory of fluctuations is obtained (in the 'thermodynamic' limit, in which $\varepsilon=E / N \Gamma_{0}^{2}=N e$ is held constant). Pointin and Lundgren's fluctuation theory, which was made explicit only for the doubly-periodic domain, was recently generalised and extended by the authors (Esler et al. 2013, EAM13 hereafter) to all simply-connected bounded two-dimensional domains $\mathcal{D} \subset \mathbb{R}^{2}$. However, cumulant expansion is laborious, and truncations of the cumulant expansion are difficult to justify rigorously. The results below, based on the central limit theorem (CLT hereafter), provide an alternative and much simpler method of recovering the key results of EAM13. More importantly, the new 
method allows EAM13's results to be extended to provide a number of predictions that can be tested against direct numerical simulations (DNS) of the point vortex system.

The rest of the paper is set out as follows. In section 2, the point vortex model is described, the uniform and microcanonical statistical ensembles to be investigated are defined, and the normal modes of variability of the system are introduced. In section 3 statistics are obtained for various quantities of interest under both the uniform and microcanonical ensembles. The critical inverse temperature and energy for condensation are obtained, the equilibrium energy spectra are found, the large energy asymptotics are derived and the connection is explained between the new results and those of meanfield theory. In section 4 the results are validated, first by comparison with DNS, and second by comparison between the predicted statistics and those obtained by statistical sampling of phase-space (following e.g. Campbell \& O'Neil 1991). Finally in section 5, the applications to both 2D classical turbulence and quantum turbulence are discussed, and conclusions are drawn.

\section{Background}

\subsection{The point vortex model}

The Hamiltonian point vortex model describes the motion in $\mathcal{D}$ of $N$ vortices of infinitesimal size with circulations $\Gamma_{i}(i=1, \ldots, N)$. Here, we will be mainly concerned with the case of a neutral vortex gas, satisfying

$$
\sum_{i=1}^{N} \Gamma_{i}=0
$$

being the system of most relevance to numerical simulations of 2D turbulence. Many of the results below can be generalised to the non-neutral case, i.e. flows with nonzero circulation, but the results are more complicated and will be reported elsewhere. The vortex locations $\boldsymbol{x}_{i}=\left(x_{i} y_{i}\right)^{T} \in \mathcal{D}$ evolve according to Hamilton's equations (e.g. Newton 2001)

$$
\Gamma_{i} \frac{\mathrm{d} x_{i}}{\mathrm{~d} t}=-\frac{\partial H}{\partial y_{i}}, \quad \Gamma_{i} \frac{\mathrm{d} y_{i}}{\mathrm{~d} t}=\frac{\partial H}{\partial x_{i}}, \quad i=1, \ldots, N
$$

where the Hamiltonian (Lin 1941a) is given by

$$
H\left(\boldsymbol{x}_{1}, \ldots, \boldsymbol{x}_{N}\right)=-\frac{1}{2} \sum_{i=1}^{N} \sum_{j=1, j \neq i}^{N} \Gamma_{i} \Gamma_{j} G\left(\boldsymbol{x}_{i}, \boldsymbol{x}_{j}\right)-\frac{1}{2} \sum_{i=1}^{N} \Gamma_{i}^{2} g\left(\boldsymbol{x}_{i}, \boldsymbol{x}_{i}\right) .
$$

Here $G\left(\boldsymbol{x}, \boldsymbol{x}^{\prime}\right)$ is the Green's function of the first kind of the Laplacian operator in $\mathcal{D}$, satisfying

$$
\nabla^{2} G\left(\boldsymbol{x}, \boldsymbol{x}^{\prime}\right)=\delta\left(\boldsymbol{x}-\boldsymbol{x}^{\prime}\right), \quad G\left(\boldsymbol{x}, \boldsymbol{x}^{\prime}\right)=0 \quad \text { on } \partial \mathcal{D},
$$


where the Laplacian $\nabla^{2}$ acts on $\boldsymbol{x}$ only, $\partial \mathcal{D}$ denotes the boundary of $\mathcal{D}$, and

$$
g\left(\boldsymbol{x}, \boldsymbol{x}^{\prime}\right)=G\left(\boldsymbol{x}, \boldsymbol{x}^{\prime}\right)-\frac{1}{2 \pi} \log \left|\boldsymbol{x}-\boldsymbol{x}^{\prime}\right| .
$$

Note that the terms involving $g$ in (2.2) only require the evaluation of the so-called Robin function $r(\boldsymbol{x})=g(\boldsymbol{x}, \boldsymbol{x})$ in which the position arguments of $g$ are set to be equal. The Robin function describes the interaction of each individual vortex with the domain boundary, for example in some domains it can be expressed as the streamfunction induced by one or more 'image' vortices outside the domain, evaluated at the location of the vortex itself. A solitary vortex in $\mathcal{D}$ will move along a path with $r(\boldsymbol{x})=$ constant.

The statistical mechanics of the system (2.1) will be discussed next.

\subsection{Microcanonical statistical mechanics}

Provided that $\mathcal{D}$ has no continuous symmetries, for example $\mathcal{D}$ is not a circle, periodic channel or a doubly periodic domain, the Hamiltonian $H$ is the only known conserved quantity of the motion. Provided ergodicity can be assumed, an aspect which will be discussed briefly in $\S 4.2$ below (see also Weiss \& McWilliams 1991), the relevant statistical ensemble for the dynamical system (2.1) is the microcanonical ensemble consisting of all vortex configurations with a fixed energy $H=E$. Hereafter, angle brackets will be used to denote the microcanonical ensemble average, e.g. $\langle\omega(\boldsymbol{x})\rangle$ for the average of the vorticity distribution

$$
\omega(\boldsymbol{x})=\sum_{i=1}^{N} \Gamma_{i} \delta\left(\boldsymbol{x}-\boldsymbol{x}_{i}\right) .
$$

Thermodynamic quantities can be obtained using the density of states function, i.e. the classical measure of the number of microstates occupying each energy shell, which for given vortex circulations $\boldsymbol{\Gamma}=\left(\Gamma_{1}, \ldots, \Gamma_{N}\right)^{T}$ is defined as

$$
W_{\boldsymbol{\Gamma}}(E)=\frac{1}{A^{N}} \int_{\mathcal{D}^{N}} \delta\left(E-H\left(\boldsymbol{x}_{1}, \ldots, \boldsymbol{x}_{N}\right)\right) \mathrm{d} \boldsymbol{x}_{1} \ldots \mathrm{d} \boldsymbol{x}_{N},
$$

where $A$ is the area of $\mathcal{D}$. A key insight (see Onsager 1949) is obtained from the fact that phase-space has a finite volume (equal to $A^{N}$ ). It follows that $W_{\Gamma}(E)$ must have a maximum at some value $E=E_{0}$, and decay to zero as $E \rightarrow \pm \infty$. A schematic illustration of $W_{\Gamma}(E)$ is given in Fig. 1. Entropy $S_{\Gamma}(E)$ and inverse thermodynamic temperature $\beta_{\boldsymbol{\Gamma}}(E)$ are formally defined from $W_{\boldsymbol{\Gamma}}(E)$ (setting Boltzmann's constant to be unity here without loss of generality),

$$
S_{\boldsymbol{\Gamma}}(E)=\log W_{\boldsymbol{\Gamma}}(E), \quad \beta_{\boldsymbol{\Gamma}}(E)=S_{\boldsymbol{\Gamma}}^{\prime}(E)=\frac{W_{\boldsymbol{\Gamma}}{ }^{\prime}(E)}{W_{\boldsymbol{\Gamma}}(E)} .
$$

It is immediately apparent from Fig. 1 that, depending on the energy $E, \beta_{\boldsymbol{\Gamma}}$ can be positive or negative. It is well-known (e.g. Onsager 1949) that the sign of $\beta_{\boldsymbol{\Gamma}}$ reveals much about the qualitative behaviour of the associated dynamics. Strongly positive $\beta_{\boldsymbol{\Gamma}}$ 


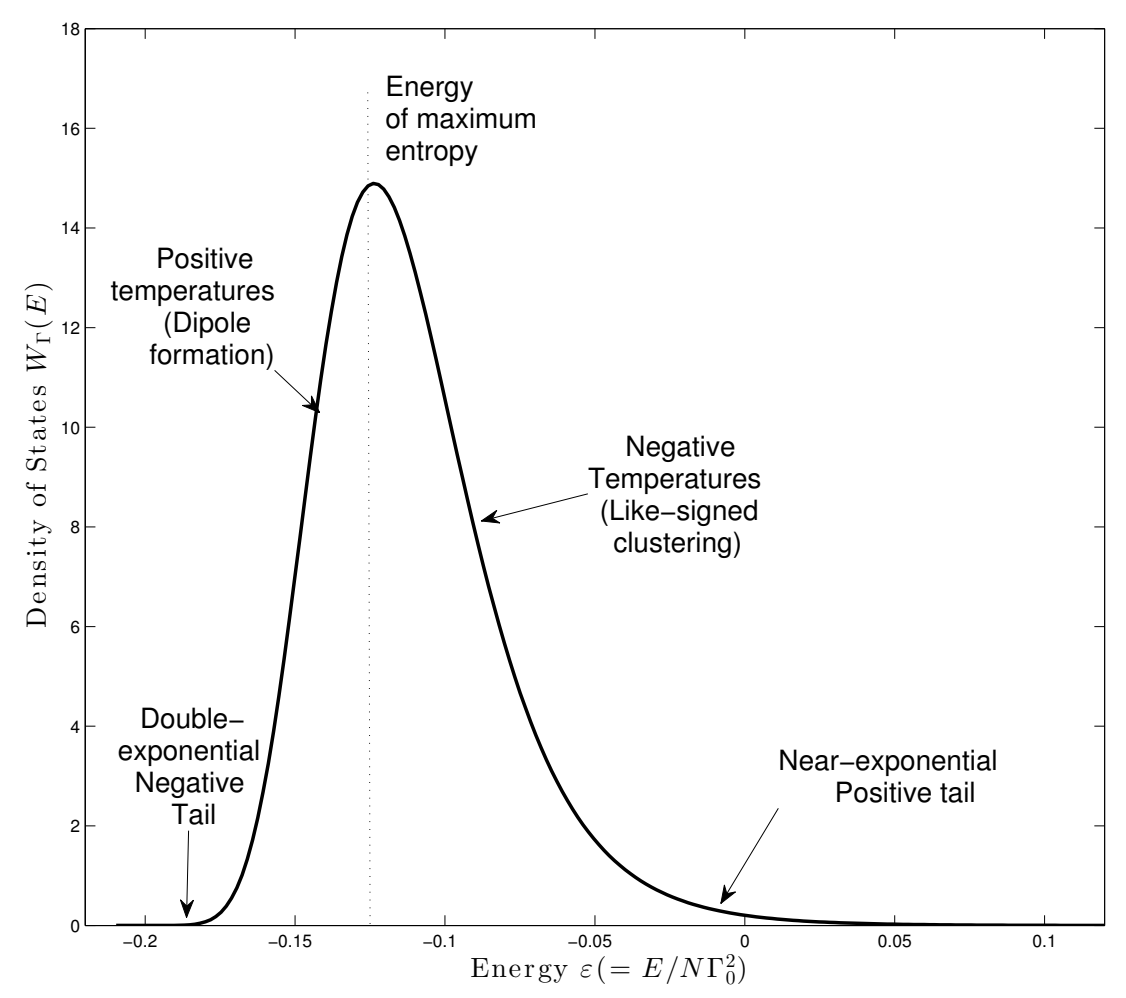

Figure 1. Schematic of the density of states function $W_{\Gamma}(E)$. (The plotted curve is in fact obtained from a numerical evaluation of $W(\varepsilon)$ for a uniform neutral gas in domain A - see $\S 3.1$.

indicates that the vortex gas will be dominated by dipoles of opposite-signed vortices, whereas strongly negative $\beta_{\boldsymbol{\Gamma}}$ indicates that clusters of like-signed vortices will form.

A useful starting point for any statistical theory is to describe the behaviour of $W_{\Gamma}(E)$ as $N \rightarrow \infty$. Note that, in this limit, it can be expected that the $N$ self-interaction terms in $(2.2)$ will be dominated by the $N(N-1)$ interactions between vortices. We will return to this point below. An important theoretical result concerning the $N \rightarrow \infty$ limit, proved by Campbell \& O'Neil (1991) for periodic, rectangular or trapezoidal domains, is that under mild restrictions on $\boldsymbol{\Gamma}$,

$$
\lim _{N \rightarrow \infty} N \Gamma_{0}^{2} W_{\Gamma}\left(N \Gamma_{0}^{2} \varepsilon\right)=W(\varepsilon) .
$$

The limiting form $W(\varepsilon)$ is a function of a rescaled energy $\varepsilon=E / N \Gamma_{0}^{2}$, which is an energy per unit vortex sometimes referred to as the thermodynamic energy. Here the 
root-mean-squared (rms) vortex circulation $\Gamma_{0}$ has the standard definition

$$
\Gamma_{0}=\left(\frac{1}{N} \sum_{i=1}^{N} \Gamma_{i}^{2}\right)^{1 / 2} .
$$

Statistical sampling of phase space, for values of $N$ in the range 10 to 160 , was used by Campbell \& O'Neil (1991) to produce convincing numerical evidence that $W_{\boldsymbol{\Gamma}}(E)$ indeed convergences according to (2.7). An important point is that (2.7) holds even if the rms circulation $\Gamma_{0}$ depends on $N$. Different authors have followed different conventions, for example in order to specify the domain-integrated vorticity of each sign, EAM13 chose $\boldsymbol{\Gamma} \sim N^{-1}$. By contrast, Dritschel et al. (2015) chose $\boldsymbol{\Gamma} \sim N^{-1 / 2}$, which has the advantage that it leads to convergence of $W_{\boldsymbol{\Gamma}}(E)$ with respect to the original energy $E$. All such scaling choices for the circulations are (trivially) equivalent to a rescaling of the time variable in (2.1) and therefore have no influence on the statistics of the system.

EAM13's main results are for the uniform neutral vortex gas, which consists of $N$ vortices exactly half having positive circulation $\Gamma_{i}=1 / N$ and half negative $\Gamma_{i}=-1 / N$. For the uniform neutral gas set-up, EAM13 used the cumulant expansion method to obtain an explicit expression for $W(\varepsilon)$, in the form of an inverse Fourier integral, generalising a result of Pointin \& Lundgren (1976) for the doubly-periodic domain. Here, we aim to obtain an analytic expression for the limiting form $W(\varepsilon)$ for as general a distribution of vortices as possible, using a new method. Not only will EAM13's results be generalised, and the scaling result (2.7) of Campbell \& O'Neil (1991) generalised to arbitrary domains, but also the new methodology will allow the explicit calculation of pdfs of various quantities in the microcanonical ensemble, which can then be verified against DNS.

\subsection{Modes of variability of an ideal two-dimensional fluid in a bounded domain}

To obtain point vortex statistics in a general domain $\mathcal{D}$ it is useful to first define an orthogonal basis that captures the modes of variability of the point vortex distribution in $\mathcal{D}$. The method to generate such a basis is to solve the hydrodynamic (e.g. Flucher \& Gustafsson 1999) eigenvalue problem for the Laplacian

$$
\nabla^{2} \Phi=\frac{\beta}{A} \Phi \text { in } \mathcal{D}
$$

subject to boundary conditions

$$
\Phi=\text { cons. on } \partial \mathcal{D}, \quad \oint_{\partial \mathcal{D}} \nabla \Phi \cdot \boldsymbol{n} \mathrm{d} s=0,
$$

Solutions of (2.9) consist of an infinite set of eigenvalues $\left\{\beta_{j}\right\}$ and associated eigenfunctions $\left\{\Phi_{j}(\boldsymbol{x})\right\}(j=0,1,2, \ldots)$. Note that $\beta$ has been deliberately chosen to label the eigenvalue parameter because the eigenvalues $\left\{\beta_{j}\right\}$ can be interpreted as inverse temperatures (see EAM13 and below). It is important to emphasize that the problem (2.9-2.10) is uniquely determined, and in general gives a different spectrum and set of basis func- 


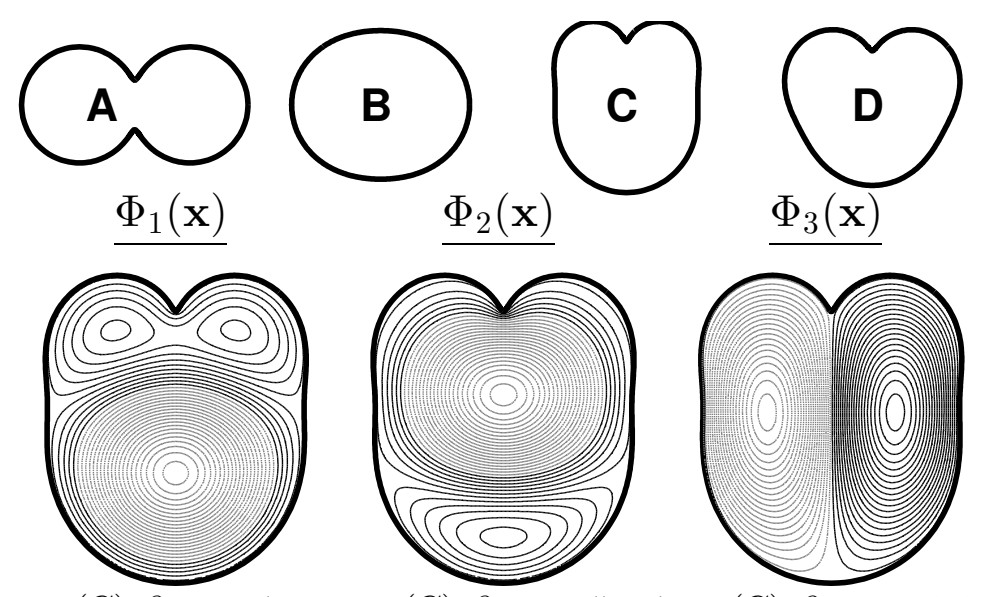
(C) $\beta_{1}=-45.13$
(C) $\beta_{2}=-47.14$
(C) $\beta_{3}=-50.89$

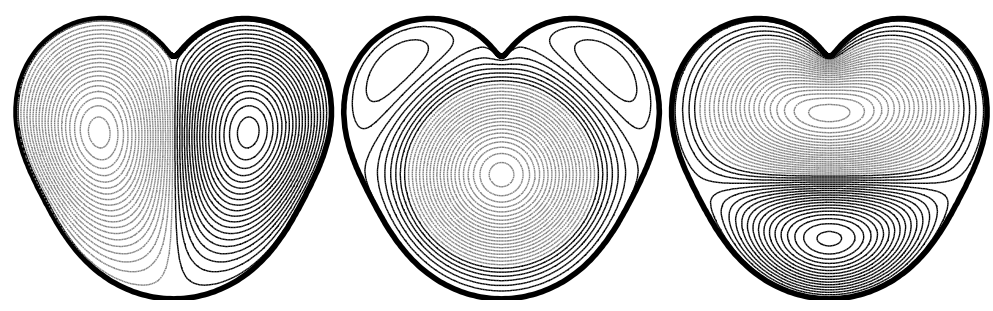

$\begin{array}{lll}\text { (D) } \beta_{1}=-44.11 & \text { (D) } \beta_{2}=-45.96 & \text { (D) } \beta_{3}=-53.69\end{array}$

Figure 2. Top Row: Domains A-D. Middle Row: Leading three eigenfunctions of domain C. Bottom row: Leading three eigenfunctions of domain D.

tions compared to the more familiar Neumann $(\nabla \Phi \cdot \boldsymbol{n}=0$ on $\partial \mathcal{D})$ and Dirichlet $(\Phi=0$ on $\partial \mathcal{D}$ ) eigenvalue problems.

The boundary condition (2.10) is natural if $\Phi$ is interpreted as a streamfunction of a vorticity field that integrates to zero in $\mathcal{D}$. In that case, because there can be no flow through $\partial \mathcal{D}, \Phi$ must be constant there, although the value of the constant need not be specified. The integral condition in (2.10) follows from the 2D divergence theorem, because the domain integral of the vorticity field $\nabla^{2} \Phi$ remains zero at all times. The lead eigenvalue $\beta_{0}$ of (2.9) is evidently zero, with corresponding eigenfunction $\Phi_{0}=$ constant in $\mathcal{D}$. An energy argument reveals that the remaining eigenvalues $\left\{\beta_{j}\right\}(j \geqslant 1)$ are strictly negative. Notice that the $1 / A$ factor in (2.9) ensures that the $\left\{\beta_{j}\right\}$ are invariant under a rescaling of the domain size. Since (2.9) satisfies the conditions for the Hilbert-Schmidt theorem (e.g. Debnath \& Mikusiński 2005), it follows that the remaining eigenfunctions $\left\{\Phi_{j}\right\}$ are orthogonal, both to each other and to $\Phi_{0}$. Using square brackets [.] to denote 
the domain average of a function $f(\boldsymbol{x})$

$$
[f]=\frac{1}{A} \int_{\mathcal{D}} f(\boldsymbol{x}) \mathrm{d} \boldsymbol{x}
$$

the eigenfunctions satisfy, following normalisation,

$$
\left[\Phi_{j}\right]=0, \quad(j \geqslant 1), \quad\left[\Phi_{j} \Phi_{k}\right]=\delta_{j k}, \quad(j, k \geqslant 1),
$$

where $\delta_{j k}$ denotes the Kronecker delta. The orthogonality properties (2.12) are of central importance to our approach below.

The relevance of the set of eigenfunctions $\left\{\Phi_{j}\right\}$ is not restricted to the point vortex system. The set $\left\{\Phi_{j}\right\}$ are in fact a natural basis for describing the variability of any ideal flow with vorticity in $\mathcal{D}$. In fact, Chavanis \& Sommeria (1996) identified the same eigenvalue problem as central to their study of the Miller-Robert-Sommeria mean-field statistical mechanics predictions (Miller 1990; Robert 1991) for the outcome of vortex patch flows in rectangular and circular domains. Their formulation of (2.9) appears rather different

$$
\nabla^{2} \Phi^{\prime}=\frac{\beta}{A}\left(\Phi^{\prime}-\left[\Phi^{\prime}\right]\right), \quad \Phi^{\prime}=0, \quad \text { on } \partial \mathcal{D},
$$

although (2.13) is easily shown to be equivalent to (2.9) under the transformation $\Phi=$ $\Phi^{\prime}-\left[\Phi^{\prime}\right]$. Taylor et al. (2009) classified the eigenfunctions of (2.13) into Type I (with $\left.\left[\Phi^{\prime}\right]=0\right)$ and Type II $\left(\left[\Phi^{\prime}\right] \neq 0\right)$. Examining the rather simpler hydrodynamic formulation of (2.9), however, this distinction is seen to be somewhat artificial in general, with the existence of Type I solutions being dependent upon symmetries of $\mathcal{D}$ (e.g. rectangles have three times as many Type I eigenfunctions as Type II).

For the purposes of comparison with DNS (see $\S 4.1$ ), and with statistics obtained by sampling of phase-space (see §4.2), four conformal domains (A-D) shown in Fig. 2, have been chosen for detailed study. These shapes may appear somewhat arbitrary, however there is a good reason for avoiding (a) the unit circle, because its statistical mechanics are complicated by the fact that angular momentum is conserved (e.g. Bühler 2002), (b) the doubly-periodic domain which conserves linear impulse (e.g. Weiss \& McWilliams 1991), and (c) rectangular domains because the Green's function then requires relatively expensive evaluations of the Weierstrass $\sigma$-function (e.g. Kunin et al. 1994). Domains A-D have the advantage that (2.1) can be transformed to the circle domain in which the Hamiltonian is closely related to that of the unit circle itself (Newton 2001).

Domains A-D are defined by a two-parameter $(q, s)$ map from the unit circle $(|Z| \leqslant 1)$,

$$
z=a Z /\left(\left(1-q^{2} Z\right)(1-\mathrm{i} s Z)\right) .
$$

Here $a=a(q, s)$ is a normalizing constant calculated to fix the area $(A=\pi)$, and $(q, s)$ for each domain are (A) $(0.8,0)$, (B) $(0.3,0),(\mathrm{C})(0.55,0.65)$ (D) $(0.55,0.5)$. When $s=0$ the map is the 'Neumann oval' (see e.g. Richardson 1981).

Fig. 2 shows the four domains (A-D) and contours the leading three eigenfunctions 
for domains C and D (for domains A and B see Figs. 3-4 of EAM13). The eigenfunction calculations are effected by transforming to the unit circle domain, and using a standard spectral method with a Chebyshev polynomial representation in the radial direction and a Fourier representation in the azimuthal direction (e.g. Chaps. 9 and 11 of Trefethen 2000, and appendix $\mathrm{C}$ of EAM13). For the results illustrated a grid of 51 radial $\times 100$ azimuthal points was used. Domains C and D exhibit an interesting switch in the ordering of eigenfunctions, the consequences of which will be explored in $\S 4.1$ below.

\subsection{The fixed ratio neutral vortex gas and its continuum limit}

The results to follow use the CLT and are therefore obtained formally by passage to a limit in which the number of vortices $N \rightarrow \infty$. For definiteness, it is helpful to be specific about how exactly the distribution of circulations $\Gamma_{i}(i=1, \ldots, N)$ of the vortices behave as the $N \rightarrow \infty$ limit is taken, while nevertheless keeping the treatment as general as possible.

With the above in mind, a 'Fixed Ratio Neutral Vortex Gas' is defined as follows. A fixed ratio $\alpha_{k}$ of the vortices is assumed to have a constant circulation $\bar{\Gamma}_{k}$, where $k=1, \ldots, K$ and $K<\infty$ is the number of different vortex populations. Evidently $\sum_{k=1}^{K} \alpha_{k}=1$. As discussed above, because of time-rescaling, the $\bar{\Gamma}_{k}$ can be constants or, provided their ratios are fixed, can multiply an arbitrary function of $N$ without affecting the argument below. The neutrality condition is then

$$
\sum_{k=1}^{K} \alpha_{k} \bar{\Gamma}_{k}=0
$$

and the rms circulation introduced above is

$$
\Gamma_{0}=\left(\sum_{k=1}^{K} \alpha_{k} \bar{\Gamma}_{k}^{2}\right)^{1 / 2} .
$$

Note that the uniform neutral vortex gas, defined in $\S 2.2$ above, is an example of a fixed ratio gas with $K=2, \alpha_{1,2}=1 / 2$ and in the treatment of EAM13, $\bar{\Gamma}_{1,2}= \pm 1 / N$.

To describe the continuum limit of the fixed ratio neutral vortex gas, we introduce $\alpha(\gamma)$ to describe the fractional density of vortices with circulation $\gamma$. A sufficient condition for our results below to hold, that will apply in all practical situations, is that vortex circulations are bounded above by a maximum value $\gamma_{m}$ (i.e. $\alpha(\gamma)=0$ for $|\gamma|>\gamma_{m}$ ). The neutrality condition and rms circulation are then

$$
\int_{-\gamma_{m}}^{\gamma_{m}} \gamma \alpha(\gamma) \mathrm{d} \gamma=0, \quad \Gamma_{0}=\left(\int_{-\gamma_{m}}^{\gamma_{m}} \gamma^{2} \alpha(\gamma) \mathrm{d} \gamma\right)^{1 / 2}
$$

The continuum case is particularly interesting because universal scaling laws have been proposed for distributions of vortex sizes in decaying two-dimensional turbulence (Dritschel et al. 2008). 


\subsection{Sums of $\chi$-squared random variables}

Many of the results below will be expressed in terms of functions which arise as a result of summations of $\chi$-squared random variables. Here, some useful results and definitions are given, which will serve to simplify the exposition below.

A starting point is the standard result that, if $X \sim \mathcal{N}(0,1)$ is a Gaussian random variable, then $Y=X^{2}-1$ is $\chi_{1}^{2}$-distributed with pdf

$$
p(y)=\left\{\begin{array}{ll}
\frac{1}{\sqrt{2 \pi}} \frac{\exp (-(y+1) / 2)}{(y+1)^{1 / 2}} & y>-1 \\
0 & y \leqslant-1
\end{array} .\right.
$$

Notice that $\mathbb{E}(Y)=0$ and $\operatorname{Var}(Y)=1$. Next suppose that we are interested in finding the pdf of the summation

$$
S_{M}=\sum_{j=1}^{M} \frac{Y_{j}}{2 w_{j}}
$$

in the limit $M \rightarrow \infty$. Here $\left\{Y_{j}\right\}$ are independent and identically distributed (iid hereafter) with distribution (2.16), and the $\left\{w_{j}\right\}$ are an infinite set of monotonically increasing positive constants. The $\left\{w_{j}\right\}$ are assumed to increase sufficiently rapidly so that (a) CLT results for weighted sums (e.g. Lyapunov CLT) do not hold, and (b) $S_{\infty}=\lim _{M \rightarrow \infty} S_{M}$ is well defined. It turns out that $w_{j} \sim j$ at large $j$ is sufficient for both (a) and (b).

Denote the pdf of $S_{M}$ by $F^{\boldsymbol{w}^{M}}(s)$ where $\boldsymbol{w}^{M}=\left(w_{1} w_{2} \ldots w_{M}\right)^{T}$. Using the convolution theorem, the Fourier transform of $F^{\boldsymbol{w}^{M}}(s)$ is given by

$$
\hat{F}^{\boldsymbol{w}^{M}}(k)=(2 \pi)^{(M-1) / 2} \prod_{j=1}^{M} \hat{p}\left(\frac{k}{2 w_{j}}\right) .
$$

where

$$
\hat{p}(k)=\frac{1}{\sqrt{2 \pi}} \frac{\exp \left(\frac{1}{2} \mathrm{i}\left(2 k-\tan ^{-1}(2 k)\right)\right)}{\left(1+4 k^{2}\right)^{1 / 4}} .
$$

is the Fourier transform of $p(y)$. Taking the limit $M \rightarrow \infty$, and using $\boldsymbol{w}$ in place of $\boldsymbol{w}^{\infty}$, after some working it follows that

$$
F^{\boldsymbol{w}}(s)=\frac{1}{2 \pi} \int_{-\infty}^{\infty} A^{\boldsymbol{w}}(k) \exp \left(\mathrm{i} k s+\mathrm{i} \phi^{\boldsymbol{w}}(k)\right) \mathrm{d} k,
$$

where the amplitude and phase functions $A^{\boldsymbol{w}}$ and $\phi^{\boldsymbol{w}}$ are real-valued and defined by

$$
A^{\boldsymbol{w}}(k)=\prod_{j=1}^{\infty}\left(1+\frac{k^{2}}{w_{j}^{2}}\right)^{-1 / 4}, \quad \phi^{\boldsymbol{w}}(k)=\frac{1}{2} \sum_{j=1}^{\infty}\left(\frac{k}{w_{j}}-\tan ^{-1}\left(\frac{k}{w_{j}}\right)\right) .
$$

The function $F^{\boldsymbol{w}}(s)$ will be used repeatedly below. A notational device we will use in several places is to write $\boldsymbol{w}\left(i_{1}, i_{2}, i_{3}\right)$ to denote the sequence $\boldsymbol{w}$ with the terms at $j=i_{1}, i_{2}, i_{3}$ omitted (e.g. $\boldsymbol{w}(1)=\left(w_{2} w_{3} \ldots\right)^{T}$ etc.).

Unfortunately, there is no known analytical method for inverting the transform (2.20), or indeed to sum (2.17) by other means (e.g. Bausch 2013, and refs. therein). As a conse- 
quence a numerical approach must be taken. Direct numerical quadrature of (2.20) using the trapezium rule, with $\boldsymbol{w}$ truncated at a few hundred terms converges fairly rapidly with element size, provided that $s \lesssim 2\left(\sum_{j} w_{j}^{-2}\right)^{1 / 2}$ (roughly four standard deviations of the resulting distribution). For larger $|s|$ convergence rapidly becomes computationally expensive and then impossible. An ingenious direct numerical pairwise method of summation of (2.17) by Bausch (2013), which is very accurate and efficient for $s<0$, has been used to cross-validate our calculations.

\section{A new approach to point vortex statistics}

\subsection{Statistics of the uniform ensemble}

A natural starting point is to formulate a description of the statistics of the uniform ensemble. The uniform ensemble pdf $p_{0}(q)$ of a quantity $Q\left(\boldsymbol{x}_{1}, \ldots, \boldsymbol{x}_{N}\right)$ that depends on the vortex positions is defined to be

$$
p_{0}(q)=\frac{1}{A^{N}} \int_{\mathcal{D}^{N}} \delta\left(Q\left(\boldsymbol{x}_{1}, \ldots, \boldsymbol{x}_{N}\right)-q\right) \mathrm{d} \boldsymbol{x}_{1} \ldots \mathrm{d} \boldsymbol{x}_{N} .
$$

In other words, $p_{0}(q)$ is the pdf of $Q$ when the vortices are arranged at random in $\mathcal{D}$ under uniform measure. Certainly, the uniform ensemble bears no relation to the statistics of quantities calculable from dynamical simulations of (2.1), which are governed by the microcanonical ensemble (see below). However, the density of states function $W_{\boldsymbol{\Gamma}}(E)$ (2.5 above), is simply the pdf of the Hamiltonian under the uniform ensemble. Other important thermodynamic quantities are defined from $W_{\Gamma}(E)$ (see eqn. 2.6). The main aim in this section is to determine the limiting behaviour of $W_{\Gamma}(E)$ as $N \rightarrow \infty$ and to establish related results that allow us to address the statistics of the microcanonical ensemble below.

The results below will be derived first for the fixed ratio neutral gas of $\S 2.4$, and the continuum limit will subsequently be taken. First define the 'vorticity projections' $\Omega_{j}$, i.e. the projections of the vorticity field onto the eigenfunction basis, to be

$$
\Omega_{j}=\frac{1}{\omega_{0}}\left[\omega \Phi_{j}\right]=\frac{1}{\omega_{0} A} \sum_{i=1}^{N} \Gamma_{i} \Phi_{j}\left(\boldsymbol{x}_{i}\right), \text { so that } \omega(\boldsymbol{x})=\omega_{0} \sum_{j=1}^{\infty} \Omega_{j} \Phi_{j}(\boldsymbol{x}) .
$$

Here $\omega_{0}=N^{1 / 2} \Gamma_{0} / A$ is a scaling factor used to simplify the bookkeeping. It is straightforward to obtain the statistics of the $\left\{\Omega_{j}\right\}$, under the uniform ensemble, using the CLT. Notice first that we can write

$$
\Omega_{j}=\sum_{k=1}^{K} \alpha_{k}^{1 / 2} \frac{\bar{\Gamma}_{k}}{\Gamma_{0}} \Omega_{j}^{k}, \quad \text { where } \quad \Omega_{j}^{k}=\frac{1}{\left(\alpha_{k} N\right)^{1 / 2}} \sum_{i_{k}=1}^{\alpha_{k} N} \Phi_{j}\left(\boldsymbol{x}_{i_{k}}\right),
$$

where the $\left\{\boldsymbol{x}_{i_{k}}\right\}$ denote the positions of those vortices with circulation $\bar{\Gamma}_{k}$. Under the uniform ensemble, each $\Omega_{j}^{k}$ is the sum of $\alpha_{k} N$ iid random variables $\Phi_{j}\left(\boldsymbol{X}_{i_{k}}\right)$, where the $\boldsymbol{X}_{i_{k}}$ are random positions uniformly distributed in $\mathcal{D}$, divided by the square root of 
the number in the sum. Applying the CLT to $\Omega_{j}^{k}$, and noting that it follows from the orthogonality properties (2.12) that $\mathbb{E}\left(\Phi_{j}\left(\boldsymbol{X}_{i_{k}}\right)\right)=0$ and $\operatorname{Var}\left(\Phi_{j}\left(\boldsymbol{X}_{i_{k}}\right)\right)=1$, it follows that in the limit $N \rightarrow \infty$ the random variable $\Omega_{j}^{k}$ is Gaussian-distributed with zero mean and unit variance, i.e. $\Omega_{j}^{k} \sim \mathcal{N}(0,1)$. The distribution for $\Omega_{j}$ then follows from the standard result for sums of Gaussian random variables applied to (3.3), from which it is also the case that $\Omega_{j} \sim \mathcal{N}(0,1)$, or equivalently the $\left\{\Omega_{j}\right\}$ have pdf

$$
p_{0}\left(\omega_{j}\right)=\frac{\exp \left(-\omega_{j}^{2} / 2\right)}{\sqrt{2 \pi}} .
$$

Further, it follows from the orthogonality condition (2.12) that $\operatorname{Cov}\left(\Omega_{j}, \Omega_{k}\right)=0$ for $j \neq k$, hence in the limit $N \rightarrow \infty$ the set of random variables $\left\{\Omega_{j}\right\}$ are iid.

Next, introduce the distributions

$$
R\left(\boldsymbol{x}, \boldsymbol{x}^{\prime}\right)=\sum_{i=1}^{N} \Gamma_{i}^{2} \delta\left(\boldsymbol{x}-\boldsymbol{x}_{i}\right) \delta\left(\boldsymbol{x}^{\prime}-\boldsymbol{x}_{i}\right), \quad S(\boldsymbol{x})=\sum_{i=1}^{N} \Gamma_{i}^{2} \delta\left(\boldsymbol{x}-\boldsymbol{x}_{i}\right) .
$$

In terms of these distributions the point vortex energy (2.2) can be written

$$
\begin{aligned}
H & =-\frac{1}{2} \int_{\mathcal{D}^{2}}\left(\left(\omega(\boldsymbol{x}) \omega\left(\boldsymbol{x}^{\prime}\right)-R\left(\boldsymbol{x}, \boldsymbol{x}^{\prime}\right)\right) G\left(\boldsymbol{x}, \boldsymbol{x}^{\prime}\right)+R\left(\boldsymbol{x}, \boldsymbol{x}^{\prime}\right) g\left(\boldsymbol{x}, \boldsymbol{x}^{\prime}\right)\right) \mathrm{d} \boldsymbol{x} \mathrm{d} \boldsymbol{x}^{\prime} \\
& =-\frac{1}{2} \int_{\mathcal{D}^{2}}\left(\omega(\boldsymbol{x}) \omega\left(\boldsymbol{x}^{\prime}\right)-R\left(\boldsymbol{x}, \boldsymbol{x}^{\prime}\right)\right) G\left(\boldsymbol{x}, \boldsymbol{x}^{\prime}\right) \mathrm{d} \boldsymbol{x} \mathrm{d} \boldsymbol{x}^{\prime}-\frac{1}{2} \int_{\mathcal{D}} S(\boldsymbol{x}) r(\boldsymbol{x}) \mathrm{d} \boldsymbol{x} .
\end{aligned}
$$

This result can be verified by inserting (2.4) and (3.5) into (3.6).

Progress can now be made by introducing the eigenfunction expansions of $R\left(\boldsymbol{x}, \boldsymbol{x}^{\prime}\right)$ and $S(\boldsymbol{x})$ as follows

$$
\begin{aligned}
R_{j k} & =\frac{1}{\omega_{0}^{2} A^{2}} \sum_{i=1}^{N} \Gamma_{i}^{2} \Phi_{j}\left(\boldsymbol{x}_{i}\right) \Phi_{k}\left(\boldsymbol{x}_{i}\right), & R\left(\boldsymbol{x}, \boldsymbol{x}^{\prime}\right) & =\omega_{0}^{2} \sum_{j=0}^{\infty} \sum_{k=0}^{\infty} R_{j k} \Phi_{j}(\boldsymbol{x}) \Phi_{k}\left(\boldsymbol{x}^{\prime}\right), \\
S_{j} & =\frac{1}{\omega_{0}^{2} A^{2}} \sum_{i=1}^{N} \Gamma_{i}^{2} \Phi_{j}\left(\boldsymbol{x}_{i}\right), & S(\boldsymbol{x}) & =\omega_{0}^{2} A \sum_{j=0}^{\infty} S_{j} \Phi_{j}(\boldsymbol{x}) .
\end{aligned}
$$

Similarly, the Green's function $G\left(\boldsymbol{x}, \boldsymbol{x}^{\prime}\right)$ and the Robin function $r(\boldsymbol{x})$ can be expanded in the eigenfunction basis

$$
\begin{aligned}
G\left(\boldsymbol{x}, \boldsymbol{x}^{\prime}\right) & =G_{00}+\sum_{j=1}^{\infty} \frac{\Phi_{j}(\boldsymbol{x}) \Phi_{j}\left(\boldsymbol{x}^{\prime}\right)}{\beta_{j}}, & G_{00} & =\frac{1}{A^{2}} \int_{\mathcal{D}^{2}} G\left(\boldsymbol{x}, \boldsymbol{x}^{\prime}\right) \mathrm{d} \boldsymbol{x} \mathrm{d} \boldsymbol{x}^{\prime}, \\
r(\boldsymbol{x}) & =\sum_{j=0}^{\infty} r_{j} \Phi_{j}(\boldsymbol{x}), & r_{j} & =\frac{1}{A} \int_{\mathcal{D}} r(\boldsymbol{x}) \Phi_{j}(\boldsymbol{x}) \mathrm{d} \boldsymbol{x} .
\end{aligned}
$$

Using (3.6-3.10) the Hamiltonian can now be expressed in terms of the projections $\left\{\Omega_{j}, R_{j j}, S_{j}\right\}$, which can be regarded as random variables under the uniform ensemble,

$$
H=\omega_{0}^{2} A^{2}\left(\varepsilon_{0}-\frac{1}{2} \sum_{j=1}^{\infty}\left(\frac{\Omega_{j}^{2}-R_{j j}}{\beta_{j}}+r_{j} S_{j}\right)\right) \quad \text { where } \quad \varepsilon_{0}=\frac{G_{00}-r_{0}}{2} .
$$

If one now writes $R_{j j}=T_{j}+1$, it is shown in the appendix that the random variables 
$T_{j}$ and $S_{j}$ can be neglected at leading order in (3.11), essentially because they are both zero mean random variables with variance $O(1 / N)$ compared to $O(1)$ for $\Omega_{j}$. Note that it was anticipated above that contributions to $H$ from self-interaction terms, i.e. the $\left\{T_{j}\right\}$ and $\left\{S_{j}\right\}$ here, would become insignificant as $N \rightarrow \infty$. On substituting $\omega_{0}=N^{1 / 2} \Gamma_{0} / A$, (3.11) is at leading order

$$
\frac{H}{N \Gamma_{0}^{2}}-\varepsilon_{0}=-\frac{1}{2} \sum_{j=1}^{\infty} \frac{\Omega_{j}^{2}-1}{\beta_{j}}, \quad \text { where } \quad \Omega_{j} \sim \mathcal{N}(0,1),
$$

and with the Gaussian random variables $\left\{\Omega_{j}\right\}$ being iid. The result (3.12) holds in the continuum limit but with $\Gamma_{0}$ given by (2.15). Alternatively, for the specific case (see $\S 2.2$ ) of the uniform neutral gas of EAM13, $\Gamma_{0}=1 / N$.

Under the uniform ensemble, the right-hand side of (3.12) is a sum of of $\chi$-squared random variables, precisely as discussed in $\S 2.5$. Introducing a scaled energy $\varepsilon=H / N \Gamma_{0}^{2}$, it is evident that this sum of random variables will have a pdf $W(\varepsilon)$ that depends only on the properties of the domain $\mathcal{D}$, through the eigenvalues $\left\{\beta_{j}\right\}$ and the domain-dependent constant $\varepsilon_{0}$, and not on the vortex circulations $\boldsymbol{\Gamma}$. From the scaling properties of random variables, in the limit $N \rightarrow \infty$, the density of states function $W_{\Gamma}(E)$, which is the pdf of $H$, satisfies $N \Gamma_{0}^{2} W_{\Gamma}\left(N \Gamma_{0}^{2} \varepsilon\right)=W(\varepsilon)$. This observation generalises the result of Campbell $\&$ O'Neil (1991) to all simply-connected domains. Furthermore, from the results of $\S 2.5$, $W(\varepsilon)$ can be given explicitly in terms of the functions defined there as

$$
W(\varepsilon)=F^{\boldsymbol{\beta}}\left(\varepsilon-\varepsilon_{0}\right)
$$

where $\boldsymbol{\beta}=\left(-\beta_{1},-\beta_{2}, \ldots\right)^{T}$ is the vector of eigenvalues of $(2.9)$.

Equation (3.13) is precisely the formula found by EAM13 for the special case of the uniform neutral gas, including evaluating the unknown constant ( $W_{0}$ in their eqn. 41). EAM13 used the (rather laborious) cumulant expansion method of Pointin \& Lundgren (1976). The function $W(\varepsilon)$ (see also Fig. 1 of EAM13) invariably resembles the schematic of Fig. 1, which in fact shows (3.13) for domain A.

A careful comparison of the shapes of the density of states prediction (3.13) is afforded by Fig. 3 (green curves), which shows the corresponding inverse temperature $\beta(\varepsilon)=$ $W^{\prime}(\varepsilon) / W(\varepsilon)$. The differences between domains are much more evident when looking at $\beta$ as opposed to $W$. In the limit $\varepsilon \rightarrow-\infty$, the behaviour is similar for both domains A and $\mathrm{B}$, and is not inconsistent with the domain-independent behaviour predicted by Edwards \& Taylor (1974), $\beta \sim \exp (-8 \pi \varepsilon)$, (see also Pointin \& Lundgren 1976). At positive energies by contrast, in the limit $\varepsilon \rightarrow \infty$ the inverse temperature $\beta \rightarrow \beta_{1}$ i.e. the first non-zero eigenvalue of (2.9), in each domain. Notice that $\beta_{1}$ differs between domain $\mathrm{A}\left(\beta_{1} \approx-36.37\right)$ and $\mathrm{B}\left(\beta_{1} \approx-42.61\right)$. It will be shown below that $\beta \rightarrow \beta_{1}$ in all simply-connected domains and that $\beta_{c}=\beta_{1}$ therefore has a special role as the critical inverse temperature for condensation referred to in the introduction. Moreover, 

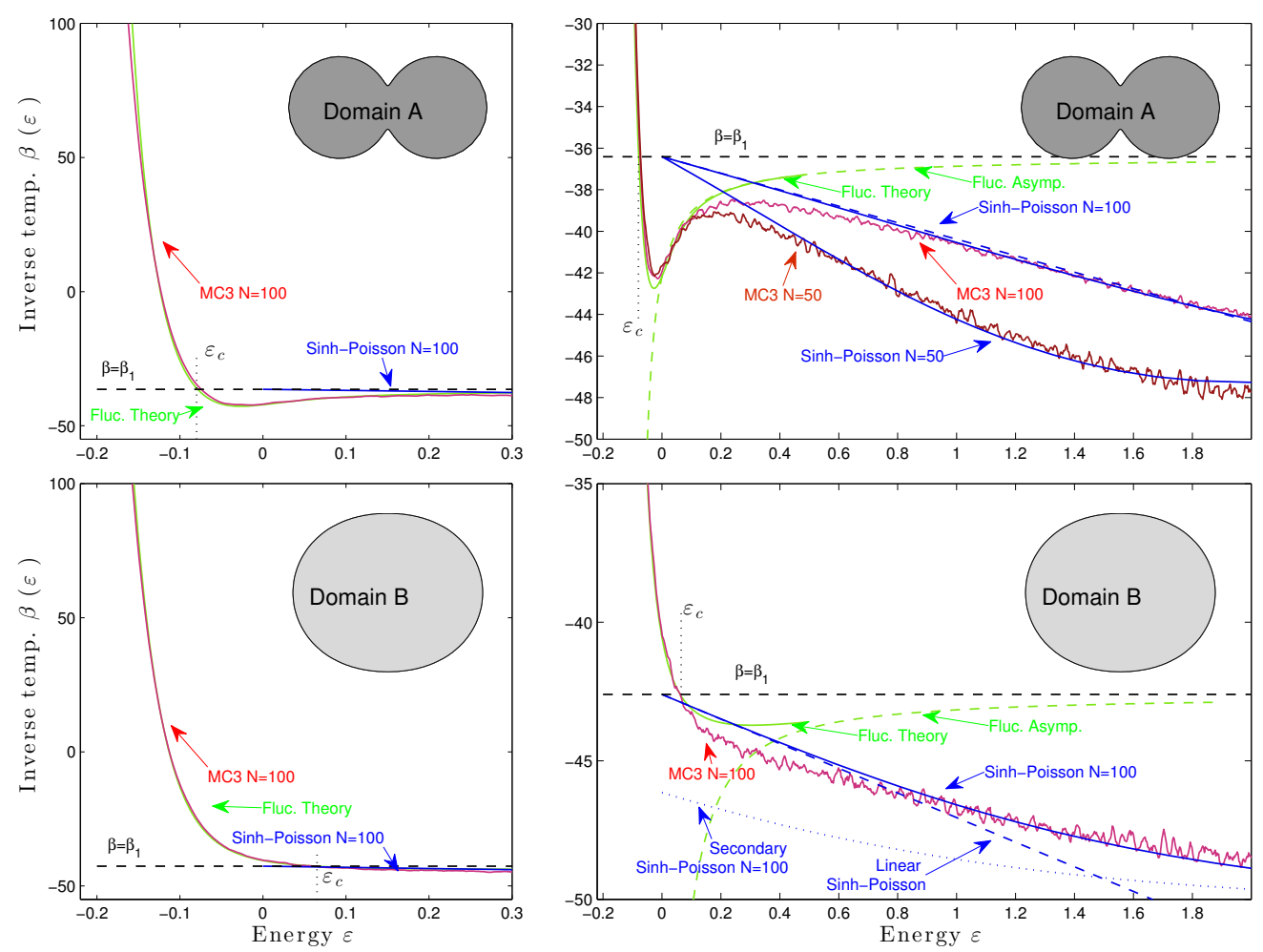

Figure 3. Comparison of inverse temperatures $\beta_{\boldsymbol{\Gamma}}(E)$ obtained from the MC3 calculation (red curves) for domains A and B (illustrated), with the theoretical result $\beta(\varepsilon)$ obtained from (3.13) (green curves), and $\beta_{h}(e)$ (see $\S 3.3$ ) obtained from the mean-field (sinh-Poisson) theory (solid blue curves). Left panels are scaled to show the central region and right panels the right-tail region of $W(\varepsilon)$. The dashed blue lines give the linearised (low $E$ ) sinh-Poisson result and the dashed green lines the large $\varepsilon$ asymptotic result (3.20). A secondary sinh-Poisson solution (dotted blue curve) is shown for domain B.

$\beta$ invariably approaches $\beta_{1}$ from below. It is interesting to remark that, in all closed domains, the point vortex microcanonical ensemble therefore has 'negative specific heat capacity' at high energies (in our notation the specific heat capacity $c$ with respect to the scaled (thermodynamic) energy $\varepsilon$ is $c=-\beta^{2} / \beta^{\prime}$, which is negative wherever $\left.\beta^{\prime}(\varepsilon)>0\right)$. Negative specific heats are generic features of long-range interacting systems and raise interesting issues concerning 'ensemble inequivalence' (e.g. Campa et al. 2009). In addition, for each domain, it is possible to define a critical energy $\varepsilon_{c}$ (vertical dotted lines on Fig. 3) to be the lowest energy (but in practice the only energy) for which $\beta\left(\varepsilon_{c}\right)=\beta_{c}$. The onset of condensation will be shown below to occur at energies close to $\varepsilon_{c}$. Fig. 3 will be discussed further below, in the context of numerical validation of the theory, when results are compared with statistics obtained by random sampling of phase-space (see $\S 4.2$ below). 
In summary, the new approach has been used here to generalise and simply the derivation of EAM13's result (3.13), showing that (3.13) is universal to all neutral distributions of vortices. Next, it will be shown that the flexibility and simplicity afforded by the current approach allows new microcanonical results to be obtained, which are directly testable by comparison with DNS.

\subsection{Statistics of the microcanonical ensemble}

The microcanonical pdf $p_{E}(q)$ of a quantity $Q\left(\boldsymbol{x}_{1}, \ldots, \boldsymbol{x}_{N}\right)$ is the distribution of $Q$ when the energy $E$ is held constant. Explicitly, this is

$$
p_{E}(q)=\frac{1}{A^{N} W_{\boldsymbol{\Gamma}}(E)} \int_{\mathcal{D}^{N}} \delta\left(Q\left(\boldsymbol{x}_{1}, \ldots, \boldsymbol{x}_{N}\right)-q\right) \delta\left(H\left(\boldsymbol{x}_{1}, \ldots, \boldsymbol{x}_{N}\right)-E\right) \mathrm{d} \boldsymbol{x}_{1} \ldots \mathrm{d} \boldsymbol{x}_{N} .
$$

Under the ergodic hypothesis, the time-series of the variable $Q(t)$ in a long-time dynamical solution of (2.1) with energy $H=E$, will be distributed as $p_{E}(q)$. However, even for moderate (i.e. $O(100)$ ) values of $N,(3.14)$ can be extremely expensive to sample numerically, particularly for energies outside the central region (although methods do exist, as will be described in $\S 4.2$ below). For many purposes (3.14) has little practical value.

By contrast, in the limit $N \rightarrow \infty$ interesting and practical microcanonical statistics can be obtained. Denoting the limiting microcanonical pdfs as $p_{\varepsilon}(q)$, where $\varepsilon=E / N \Gamma_{0}^{2}$ is the scaled energy, the techniques of $\$ 3.1$ can be adapted to make predictions which can be compared with integrations of (2.1). Here the focus will be on the microcanonical pdfs of the vorticity projections $\Omega_{j}$, which reveal how energy is shared between the normal modes of the system in the long-time limit. Recall that, under the uniform ensemble, the $\left\{\Omega_{j}\right\}$ are iid with Gaussian distribution $\mathcal{N}(0,1)$. Under the microcanonical ensemble, their pdf will deviate from Gaussianity due to the need to satisfy the energy constraint $H=N \Gamma_{0}^{2} \varepsilon$ (see eqn. 3.12).

The results are obtained using Bayes' theorem. Writing the microcanonical marginal pdf of $\Omega_{j}$ as $p_{\varepsilon}\left(\omega_{j}\right)=p_{0}\left(\omega_{j} \mid \varepsilon\right)$, and applying Bayes' theorem

$$
p_{\varepsilon}\left(\omega_{j}\right)=\frac{p_{0}\left(\varepsilon \mid \Omega_{j}=\omega_{j}\right) p_{0}\left(\omega_{j}\right)}{p_{0}(\varepsilon)}=\frac{F^{\boldsymbol{\beta}(j)}\left(\varepsilon-\varepsilon_{0}+\frac{\omega_{j}^{2}-1}{2 \beta_{j}}\right)}{F^{\boldsymbol{\beta}}\left(\varepsilon-\varepsilon_{0}\right)} \frac{\exp \left(-\omega_{j}^{2} / 2\right)}{\sqrt{2 \pi}}
$$

gives the result in terms of the functions defined in $\S 2.5$ (recall that $\boldsymbol{\beta}(j)$ denotes the vector $\boldsymbol{\beta}$ with the $j$ th term omitted). The conditional probability in the numerator of (3.15) is expressed in terms of the function $F^{\boldsymbol{\beta}(j)}(\cdot)$ because the latter is the pdf for the sum of random variables in (3.12) excluding the $j$ th term, and its argument follows from the fact that total sum in (3.12) must remain equal to $\varepsilon-\varepsilon_{0}$ in the case where $\Omega_{j}=\omega_{j}$ is given.

The pdf $p_{\varepsilon}\left(\omega_{1}\right)$ of the first vorticity projection $\Omega_{1}$ deviates most strongly from the Gaussian obtained for the uniform ensemble. In Fig. 4 (top panels) $p_{\varepsilon}\left(\omega_{1}\right)$, calculated 

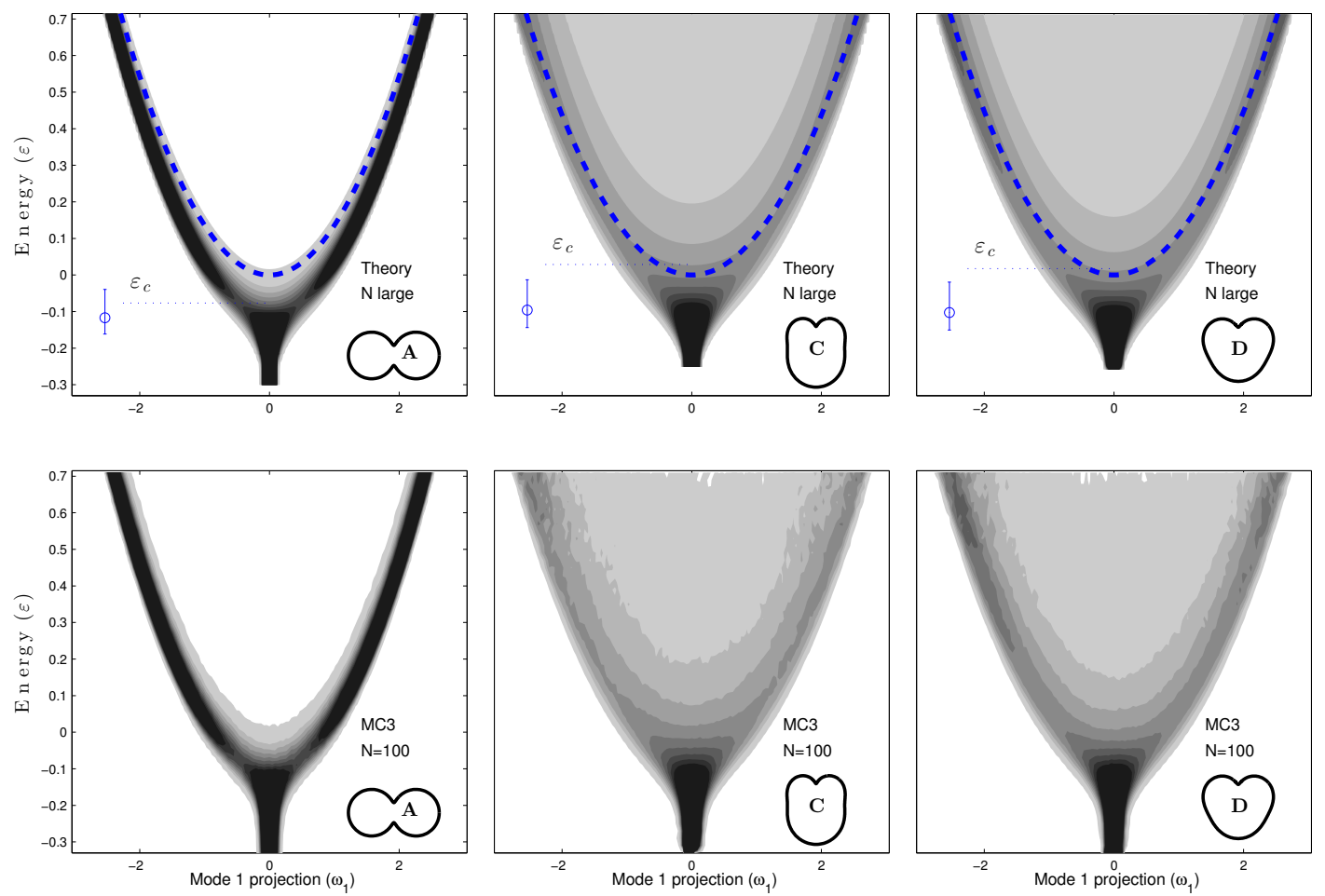

Figure 4. Upper panels: Contour plots of $p_{\varepsilon}\left(\omega_{1}\right)$, from the theoretical expression (3.15), as a function of $\left(\omega_{1}, \varepsilon\right)$ for domains A, C and D. The blue circle and error bars denote the mean and $95 \%$ confidence intervals of $W(\varepsilon)$ and the critical energy $\varepsilon_{c}$ is marked with a dotted line. The possible values of $\omega_{1}$ according to the linearised sinh-Poisson solution (3.28) are marked with the dashed blue parabolas. Lower panels: As for the upper panels, but here $p_{\varepsilon}\left(\omega_{1}\right)$ is estimated using MC3 sampling of phase-space with $N=100$ vortices.

using eqn. (3.15), is contoured as a function of $\left(\omega_{1}, \varepsilon\right)$ for domains A, C and D. All three domains share a key feature. At energies around the level $\varepsilon=\varepsilon_{c}$ the pdf switches from unimodal to bimodal, and the distribution becomes increasing bimodal as the energy is further increased. A strongly bimodal distribution can be associated with a state with a coherent mean flow (i.e. the condensed state), because in this case there will be a strong tendency for the system to remain either in a positive polarity state with $\Omega_{1}>0$ or in a negative polarity state with $\Omega_{1}<0$. Passage between the two states requires the system to evolve through a relatively improbable configuration with $\Omega_{1}=0$ (similar transitions between positive and negative polarity equilibrium solutions of the Euler equations have been studied by Naso et al. 2010).

In Fig. 4, domain A is seen to have a very 'clean' condensation, in the sense that $p_{\varepsilon}\left(\omega_{1}\right)$ becomes strongly bimodal at relatively low energies. There is a sharp contrast, which arguably might be expected on geometric grounds, between domain A and domains B-D for 

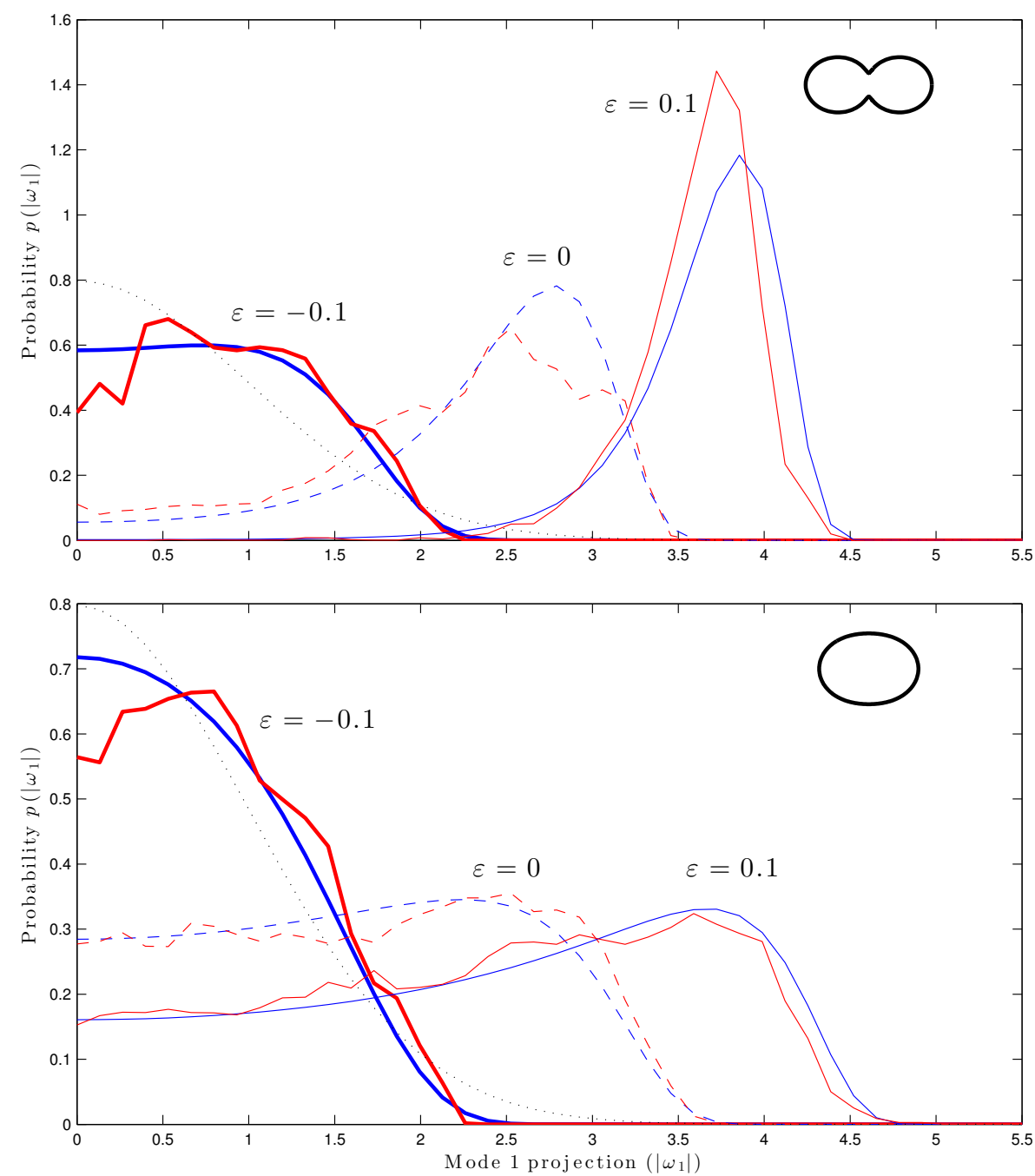

FiguRE 5. Comparison between theoretical predictions (eqn. 3.15, blue curves) of $p_{E}\left(\left|\omega_{1}\right|\right)$ and DNS (red curves) of (2.1). Three different energy levels $(\varepsilon=-0.1,0,0.1)$ are shown. (2.1). Top: Domain A. Bottom: Domain B.

which the onset of bimodality as $\varepsilon$ is increased is more gradual. In domains B-D the probability of a configuration associated with a switch in polarity $\left(\Omega_{1}=0\right)$ is much greater than that at the same energy in domain A. The issue of how the transition probability is controlled by the domain shape is discussed in the next subsections where asymptotics at high $\varepsilon$ are considered. Domains $\mathrm{C}$ and D, which have similar shapes, unsurprisingly are seen in Fig. 4 to have similar pdfs. The reason they have been chosen for close examination is that the condensate structure $\Phi_{1}(\boldsymbol{x})$ (plotted in Fig. 2) is nevertheless very different between the two domains. 

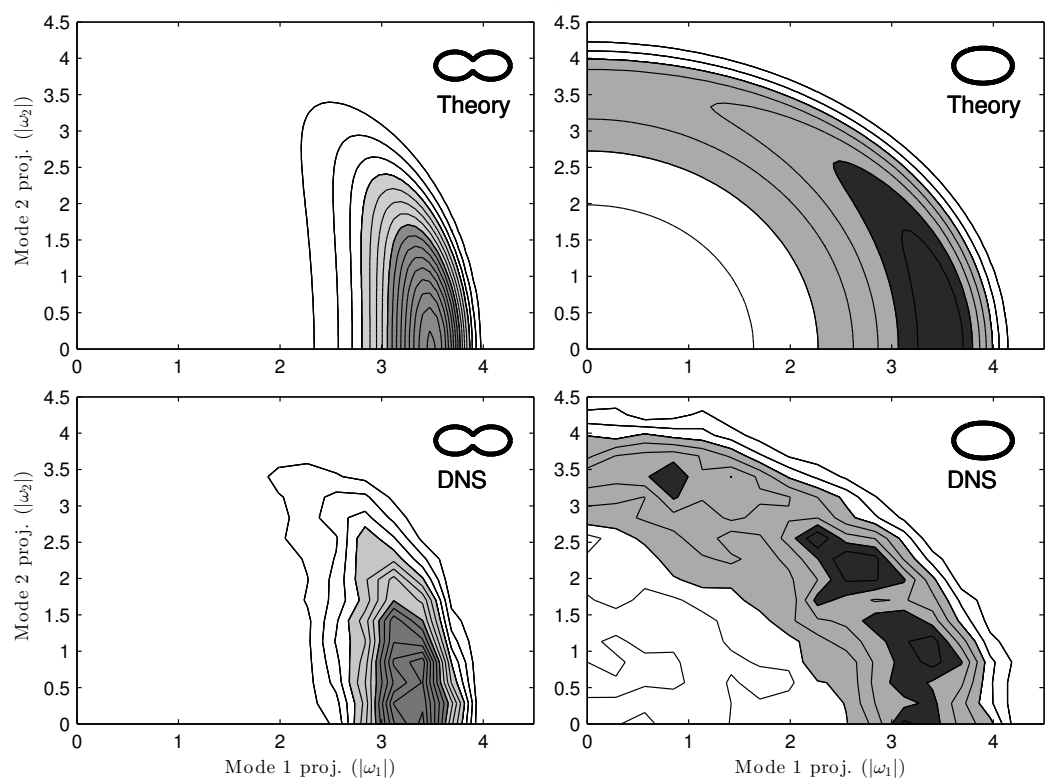

FIgURE 6. Contour plots comparing $p_{\varepsilon}\left(\left|\omega_{1}\right|,\left|\omega_{2}\right|\right)$ (upper panels) predicted by the theory (eqn. 3.16) with (lower panels) statistics compiled from the DNS of eqn. (2.1). The energy is fixed at $\varepsilon=0.05$. Left panels: Domain A. Right panels: Domain B.

The difference in the pdfs of $\Omega_{1}$ can be seen clearly in Fig. 5, where $p_{\varepsilon}\left(\omega_{1}\right)$ (blue curves) is plotted at three different values of the energy $(\varepsilon=-0.1,0,0.1)$ for domains A and B. Note that, since the pdf is an even function of $\omega_{1}$, it is plotted as a function of $\left|\omega_{1}\right|$ in Fig. 5 in order to facilitate comparison with the DNS (red curves, discussed in $\S 4.1$ below). For reference, the (Gaussian) uniform ensemble pdf is also plotted (dotted black curve).

For any ordered set of $n$ integers $\left\{i_{1}, i_{2}, \ldots, i_{n}\right\}$ the joint microcanonical pdf $p_{\varepsilon}\left(\omega_{i_{1}}, \omega_{i_{2}}, \ldots, \omega_{i_{n}}\right)$ of the random vector of vorticity projections $\left(\Omega_{i_{1}}, \Omega_{i_{2}}, \ldots, \Omega_{i_{n}}\right)^{T}$ can also be calculated. The result is

$$
p_{\varepsilon}\left(\omega_{i_{1}}, \omega_{i_{2}}, \ldots, \omega_{i_{n}}\right)=\frac{F^{\boldsymbol{\beta}\left(i_{1}, i_{2}, \ldots, i_{n}\right)}\left(\varepsilon-\varepsilon_{0}+\sum_{i_{k}} \frac{\omega_{i_{k}}^{2}-1}{2 \beta_{i_{k}}}\right)}{F^{\boldsymbol{\beta}}\left(\varepsilon-\varepsilon_{0}\right)} \frac{\exp \left(-\sum_{i_{k}} \frac{\omega_{i_{k}}^{2}}{2}\right)}{(2 \pi)^{n / 2}} .
$$

Of these joint pdfs, the most interesting is arguably $p_{\varepsilon}\left(\omega_{1}, \omega_{2}\right)$ because it illustrates the distribution of energy between the leading two normal modes of the system. Fig. 6 contours $p_{\varepsilon}\left(\omega_{1}, \omega_{2}\right)$ for domains A and B for energy $\varepsilon=0.05$. The results show markedly different behaviours for the two domains. In domain A nearly all states have a significant amount of energy trapped in mode 1, whereas domain B allows energy to be shared comparatively efficiently between modes 1 and 2 , evidenced by the circular pattern (only the upper right quadrant is shown) in Fig. 6. The difference can be explained by the 
difference in the spectral gap $\beta_{1}-\beta_{2}$, between the two domains, as will be discussed further below.

\subsection{The energy spectrum}

For the two-dimensional Euler equations in $\mathcal{D}$, it is straightforward to define the ensembleaveraged fluid dynamical energy $E_{F}$ and the associated instantaneous discrete energy spectrum. Expanding the vorticity field $\omega(\boldsymbol{x})$ (here assumed to have zero-mean) in the eigenfunctions $\left\{\Phi_{j}(\boldsymbol{x})\right\}$ as in (3.2) leads to

$$
E_{F}=-\frac{1}{2} \int_{\mathcal{D}^{2}}\left\langle\omega(\boldsymbol{x}) \omega\left(\boldsymbol{x}^{\prime}\right)\right\rangle G\left(\boldsymbol{x}, \boldsymbol{x}^{\prime}\right) \mathrm{d} \boldsymbol{x} \mathrm{d} \boldsymbol{x}^{\prime}=\omega_{0}^{2} A^{2} \sum_{j=1}^{\infty}\left\langle E_{j}\right\rangle, \text { where } E_{j}=-\frac{\Omega_{j}^{2}}{2 \beta_{j}},
$$

and $\Omega_{j}=\left[\omega \Phi_{j}\right] / \omega_{0}$ as in (3.2). In general $\omega_{0}$ is any scaling factor and the angle brackets denote an (unspecified) ensemble average. In the singular limit of the point vortex distribution (2.4), however, $E_{F}$ is undefined. Consequently, additional regularising terms are needed in the point vortex Hamiltonian (2.2), compare for example (3.6) to (3.17). Nevertheless, the fluid dynamical spectral coefficients $E_{j}$ in (3.17), which denote the energy in a mode with wavenumber $k_{j}=\left(-\beta_{j} / A\right)^{1 / 2}$, do remain calculable in the point vortex system. In this case $\omega_{0}=N^{1 / 2} \Gamma_{0} / A$ and the angle brackets unambiguously denote the microcanonical ensemble. In fact, it is demonstrated in the appendix that $\left\langle E_{j}\right\rangle$ has the following exact analytic relationship with the density of states function,

$$
\left\langle E_{j}\right\rangle=-\frac{1}{2 \beta_{j}}\left(1+\frac{\beta(\varepsilon)}{\beta_{j}}-2 \frac{\beta_{j}}{W(\varepsilon)} \frac{\partial W}{\partial \beta_{j}}(\varepsilon)\right) .
$$

It is interesting to remark that the density of states function $W(\varepsilon)$ encodes, through (3.18), all information concerning the equilibrium point vortex energy spectrum (recall that $\beta(\varepsilon)=W^{\prime}(\varepsilon) / W(\varepsilon)$ ). An alternative expression (compare eqn. 38 of EAM13), which shows that it is not in fact necessary to evaluate the partial derivatives $\partial W / \partial \beta_{j}$ to obtain the energy spectrum from $W(\varepsilon)$, is

$$
\left\langle E_{j}\right\rangle=\frac{1}{2 W(\varepsilon)} \int_{-\infty}^{\varepsilon} W(\bar{\varepsilon}) \mathrm{e}^{\beta_{j}(\varepsilon-\bar{\varepsilon})} \mathrm{d} \bar{\varepsilon} .
$$

Equation (3.19) is obtained by integrating equation (A 8) given in the appendix.

In Fig. 7 the energy spectrum obtained from (3.18) is plotted for domain B for several values of $\varepsilon$. The usual convention of summing the contributions from modes within wavenumber shells of finite width $\Delta k$ is adopted. Here we take $\Delta k=2 \sqrt{\pi}$ (consistent with taking $\Delta k=1$ in the usual doubly-periodic domain which has greater area by a factor of $4 \pi$ ). Fig. 7 shows that as $\varepsilon$ is increased, energy is increasingly added to the largest scales in the system. At small scales the spectrum has a $k^{-1}$ slope for all values of $\varepsilon$, consistent with the spectrum not being integrable. These results are consistent with spectra recently calculated numerically by Dritschel et al. (2015) for the particular case 


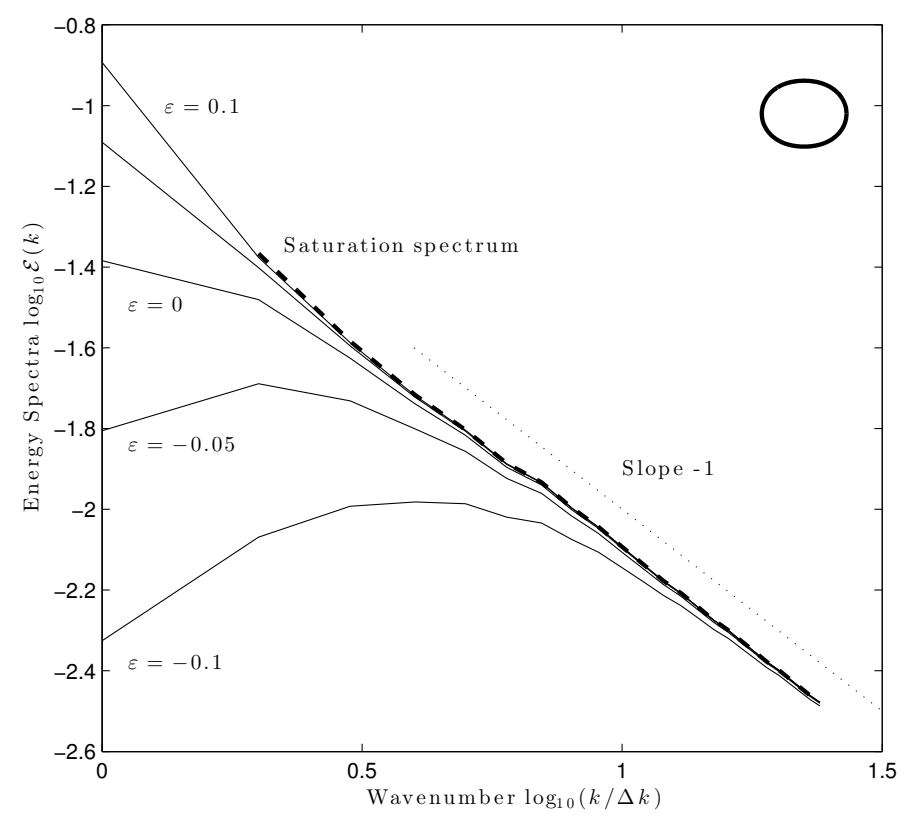

Figure 7. Point vortex energy spectra $\mathcal{E}(k)$ in domain B at different values of $\varepsilon$.

of point vortices on the sphere. The dashed curve, showing the saturated spectrum, will be discussed below.

\subsection{Large $\varepsilon$ asymptotics, condensation and saturation spectrum}

In the appendix it is proved that as $\varepsilon \rightarrow \infty$ the limiting density of states function satisfies

$$
W(\varepsilon) \approx\left(\frac{-\beta_{1}}{\pi\left(\varepsilon-\varepsilon_{0}\right)}\right)^{1 / 2} \exp \left(\beta_{1}\left(\varepsilon-\varepsilon_{0}\right)-\frac{C_{1}}{2}-\sum_{k=1}^{\infty} \frac{B_{k+1}}{k\left(\varepsilon-\varepsilon_{0}\right)^{k}}\right)
$$

where $C_{1}$ is a constant

$$
C_{1}=\sum_{j=2}^{\infty} \log \left(1-\frac{\beta_{1}}{\beta_{j}}\right)+\frac{\beta_{1}}{\beta_{j}},
$$

and the $\left\{B_{k}\right\}$ are a sequence of negative constants also depending upon the eigenvalues $\left\{\beta_{j}\right\}$ (see table 1 in the appendix). Not only does (3.20) serve as a test of the numerical quadrature of (3.13), it also allows simplified analytical expressions to be obtained for various quantities of interest. The useful domain of validity of (3.20) can be estimated by inspection of Fig. 3 (right panels), where the corresponding four term approximation $\beta(\varepsilon) \approx \sum_{k=0}^{3} B_{k}\left(\varepsilon-\varepsilon_{0}\right)^{-k}$ (see table 1 ) is plotted (dashed green curves) against a quadrature of the exact expression (i.e. obtained from eqn. 3.13, solid green curves). Evidently the approximation is appropriate only for inverse temperatures below the critical value (i.e. $\beta<\beta_{1}$ ), and works better in domain A than domain B because the coefficients $\left\{B_{k}\right\}$ decay more rapidly in the former case. 
Naturally, equation (3.20) also gives the $s \rightarrow \infty$ asymptotics of the functions $F^{\boldsymbol{w}}(s)$ of $\S 2.5$, hence essentially the same asymptotics can be applied to the microcanonical pdf $p_{\varepsilon}\left(\omega_{j}\right)$ (given by 3.15 ) of the vorticity projection $\Omega_{j}$. It is found that, as $\varepsilon \rightarrow \infty$, all but the first vorticity projection becomes normally distributed

$$
\Omega_{j} \sim \mathcal{N}\left(0, \frac{\beta_{j}}{\beta_{j}-\beta_{1}}\right) \text { for } j \geqslant 2 .
$$

Applying the method to (3.16) it can also be established that $\left\{\Omega_{2}, \Omega_{3}, \ldots\right\}$ become independent as $\varepsilon \rightarrow \infty$. Interestingly, (3.21) is consistent with saturation of the fluid dynamical energy in each mode

$$
\left\langle E_{j}\right\rangle=-\frac{\left\langle\Omega_{j}^{2}\right\rangle}{2 \beta_{j}} \rightarrow \frac{1}{2\left(\beta_{1}-\beta_{j}\right)}, \text { as } \varepsilon \rightarrow \infty, \quad \text { for } j \geqslant 2 .
$$

In other words, at large $\varepsilon$, the energy spectrum of the point vortex system becomes saturated and as $\varepsilon$ increases further, all of the additional energy must go into the condensate only. The saturated spectrum (3.22) is plotted on Fig. 7 (dashed curve). Note that, because $\left\langle E_{1}\right\rangle$ depends upon on the system energy $\varepsilon$, the first energy shell is omitted. Spectral saturation is seen to be more-or-less complete by $\varepsilon=0.1$, indicating that as $\varepsilon$ increases beyond this value all energy will go into the first energy shell (i.e. the condensate).

The pdf $p_{\varepsilon}\left(\omega_{1}\right)$ of the condensate vorticity projection $\Omega_{1}$, by contrast, does not become Gaussian as $\varepsilon \rightarrow \infty$. However, results about its behaviour can be found by taking the microcanonical ensemble average of (3.12), and using the results above. For example, the ensemble average of the condensate energy $E_{1}$ is found to be

$$
\left\langle E_{1}\right\rangle=\varepsilon-\varepsilon_{0}^{*}, \quad \varepsilon_{0}^{*}=\varepsilon_{0}+\frac{1}{2 \beta_{1}}+\sum_{j=2}^{\infty} \frac{\beta_{1}}{2 \beta_{j}\left(\beta_{1}-\beta_{j}\right)} .
$$

The domain-dependent constant $\varepsilon_{0}^{*}$ represents a correction to the mean field theory to be discussed in the next subsection. Similarly, the variance of the condensate energy about its mean value is found to be

$$
\left\langle\left(E_{1}-\left\langle E_{1}\right\rangle\right)^{2}\right\rangle=\frac{1}{4} \sum_{j=2}^{\infty} \frac{1}{\left(\beta_{1}-\beta_{j}\right)^{2}},
$$

showing that the amplitude of fluctuations of the condensate energy are independent of $\varepsilon$ as $\varepsilon \rightarrow \infty$. The predicted variance in (3.24) varies greatly between domains, e.g. $\left\langle\left(E_{1}-\left\langle E_{1}\right\rangle\right)^{2}\right\rangle \approx 7.2 \times 10^{-4}$ in domain A versus $2.4 \times 10^{-2}$ in domain $\mathrm{B}$, with the large difference explained by the clustering of the first few eigenvalues close to $\beta_{1}$ in domain $\mathrm{B}$.

Figs. 4-6 suggest that, as energy increases, switches in the sign of $\Omega_{1}$ will become increasing infrequent in the dynamics, as the probability $p_{\varepsilon}\left(\omega_{1}=0\right)$ of encountering a transition state with $\Omega_{1}=0$ becomes increasingly small. One might in fact anticipate that the expected time of transition is inversely proportional to $p_{\varepsilon}\left(\omega_{1}=0\right)$, this is in fact the case with stochastic models of bistable systems (e.g. Naso et al. 2010), which 
might be used as a simple phenomenological models for the dynamic evolution of $\Omega_{1}(t)$ here (see e.g. section 6.2 of Touchette 2009). This idea will be explored in more detail in future work. In the large $\varepsilon$ limit,

$$
p_{\varepsilon}\left(\omega_{1}=0\right) \approx\left(\frac{\beta_{2}}{\beta_{1}}\right)^{1 / 2} \exp \left(-\left(\beta_{1}-\beta_{2}\right)\left(\varepsilon-\varepsilon_{0}\right)+\frac{C_{1}-C_{2}}{2}-\frac{\beta_{2}}{2 \beta_{1}}\right)
$$

where $C_{2}$ is defined exactly as $C_{1}$ above, but with $\beta_{2}$ replacing $\beta_{1}$ and the sum starting at $j=3$. The result (3.25) shows that the frequency of switches in the sign of $\Omega_{1}$ is highly sensitive to the spectral gap $\beta_{1}-\beta_{2}>0$. In domain $\mathrm{A}, \beta_{1}-\beta_{2} \approx 33.78$, whereas in domain $\mathrm{B}, \beta_{1}-\beta_{2} \approx 3.54$, which explains the huge difference in switching behaviour between the two domains. The important point is that it is the shape of the domain that controls the spectral gap, and through this the switching frequency at high energy.

\subsection{The connection with mean-field theory (sinh-Poisson equation)}

Many researchers will be more familiar with the mean-field statistical mechanics theory of Joyce \& Montgomery (1973) than with the fluctuation theory considered above. What is the connection between the two theories? To answer this question, we will briefly review the mean-field theory for a uniform neutral gas below, and show how the main predictions relate to those of the fluctuation theory. The essential starting point is to recognise that, while the results of the fluctuation theory are expressed as a function of the scaled (thermodynamic) energy $\varepsilon=E /\left(N \Gamma_{0}^{2}\right)$, the mean-field results are expressed as a function of $e=E /\left(N^{2} \Gamma_{0}^{2}\right)$ (sometimes referred to as the hydrodynamic energy). The fact that the energy scales differently with the number of vortices $N$ in each theory $(\varepsilon=N e)$ means that, with respect to the density of states function in Fig. 1, the fluctuation theory is a theory of the central region, whereas the mean-field theory is theory of the (positive) tail region. Pushing an analogy with sums of random variables in elementary statistics, it might be said that the fluctuation theory is the 'central limit theorem' for point vortices, with the mean-field theory acting as a 'large-deviation theory'. According to this analogy, the two theories will be linked asymptotically, with the $\varepsilon \rightarrow \infty$ limit of the fluctuation theory matching to the $e \rightarrow 0$ limit of the mean-field theory (Pointin \& Lundgren 1976).

The mean field theory is formulated on the basis that the hydrodynamic energy $e$ of the system is positive, that all of this energy is contained in a steady mean flow (the condensate), and that fluctuations can be neglected. For the case of a uniform neutral gas, the mean-field theory leads to the well-known sinh-Poisson equation (Joyce \& Montgomery 1973) for the time-mean streamfunction $\psi\left(\right.$ scaled so that $\left.\nabla^{2} \psi=\langle\omega\rangle / N \Gamma_{0}\right)$ of the condensate

$$
\nabla^{2} \psi=C \sinh \left(\beta_{h} \psi\right), \text { where } C=\frac{1}{A}\left[\exp \left(\beta_{h} \psi\right)\right]^{-1 / 2}\left[\exp \left(-\beta_{h} \psi\right)\right]^{-1 / 2},
$$

where as above [.] denotes the domain average. The correct boundary condition for $\psi$ on $\partial \mathcal{D}$ is (2.10). This can be seen, for example, by following the derivation of Pointin \& 
Lundgren (1976, see discussion surrounding their eqn. 23). We note in passing that the Dirichlet condition $(\psi=0$ on $\partial \mathcal{D})$ has occasionally been erroneously used in previous works (following e.g. Book et al. 1975). That (2.10) physically makes sense is clear: $\psi=$ constant on $\partial \mathcal{D}$ is sufficient to satisfy no-normal flow, and the circulation condition on $\psi$ in (2.10) must hold from Green's theorem in the plane, because the domain integral of the mean-field vorticity $\nabla^{2} \psi$ is zero for a neutral gas. Consider also that (3.26) is not invariant under the transformation $\psi \rightarrow \psi+$ constant, therefore specifying a value for $\psi$ on the boundary by using the Dirichlet boundary condition, is evidently overprescriptive. The inverse temperature $\beta_{h}=\beta_{h}(e)$ appearing in (3.26) is determined from the mean-field energy

$$
e=-\frac{1}{2} \int_{\mathcal{D}} \psi \nabla^{2} \psi \mathrm{d} \boldsymbol{x} .
$$

It has been proved (Kiessling 1995) that $\beta_{h}(e)$ is strictly negative. As discussed above, however, it is clear that both positive and negative temperatures exist in the point vortex system. At the outset it is thus clear that the mean-field theory is restricted to a limited range of $\beta$.

Asymptotic matching with the fluctuation theory requires that the system (3.26-3.27) be studied in the limit $E \rightarrow 0$. In this limit (3.26) can be linearised to recover the eigenvalue problem (2.9). In other words, at low energies, distinct solutions of the sinhPoisson equation emerge from $\left(e, \beta_{h}\right)=\left(0, \beta_{1}\right),\left(0, \beta_{2}\right), \ldots$. The mean-field theory can be used to show (Chavanis \& Sommeria 1996; Taylor et al. 2009) that, of these solutions, the first has maximum entropy and will therefore be selected by the dynamics. In the $E \rightarrow 0$ limit the first solution has streamfunction

$$
\psi(\boldsymbol{x})= \pm\left(-\frac{2 e}{\beta_{1}}\right)^{1 / 2} \Phi_{1}(\boldsymbol{x}) .
$$

Further, Taylor et al. (2009) have shown that the caloric curve $\beta_{h}(e)$ associated with this first solution has expansion

$$
\beta_{h}(e)=\beta_{1}-\beta_{1}^{2}\left(1-\frac{1}{3}\left[\Phi_{1}^{4}\right]\right) e+O\left(e^{2}\right) .
$$

Numerical solutions of (3.26-3.27) have been obtained, using the algorithm of McDonald (1974), in domains A and B for a range of energies starting close to $E=0$. These solutions have been used to obtain numerical estimates of $\beta_{h}(e)$ for each domain, and these have been plotted on Fig. 3 (blue curves) for $N=50$ and $N=100$ vortices (note that the number of vortices $N$ affects the scaling of the $\beta_{h}(e)$ curve in Fig. 3 because the ordinate is $\varepsilon=N e$ and not $e$ ). The shape of the $\beta_{h}(e)$ curves can be seen only in the right panels, which expand the region near $\beta_{1}$. For domain $\mathrm{B}$ solutions emerging from both $\left(0, \beta_{1}\right)$ and $\left(0, \beta_{2}\right)$ (dotted line) are plotted. The corresponding small-e approximation (3.29) has also been calculated and plotted (dashed blue line).

The results above allow us to consider matching between the $\varepsilon \rightarrow \infty$ limit of the 
fluctuation theory and the $e \rightarrow 0$ limit of the mean-field theory. First note that it is proved above, and is clear from Fig. 3, that both $\lim _{\varepsilon \rightarrow \infty} \beta(\varepsilon)=\beta_{1}$ and $\lim _{e \rightarrow 0} \beta_{h}(e)=\beta_{1}$. Hence $\beta_{c}=\beta_{1}$ is not only the critical inverse temperature for condensation, but is also the critical value for matching between the theories. Therefore, as $E$ is increased at finite $N$, a smooth transition between the behaviour predicted by each theory can be expected for $\beta$ near $\beta_{c}$. This transition will be examined numerically in $\S 4.2$ below using phasespace sampling. Second, the solution (3.28) is consistent with the $\varepsilon \rightarrow \infty$ prediction for the fluctuation theory, because in the fluctuation theory all of the excess energy ends up in the condensate. The results (3.23-3.24) go beyond the mean-field theory result (3.28) by calculating the size of both the leading correction to the mean energy of the condensate and the variance of the condensate energy about this mean. The vorticity projection predicted by (3.28) is plotted on Fig. 4 as a blue dashed parabola, and shows that mean-field theory slightly underestimates the magnitude of the vorticity projections.

\section{Numerical Validation}

\subsection{Direct numerical simulation (DNS)}

The ergodic hypothesis motivating the study of the microcanonical ensemble in $\S 3.2$ is that the statistics of the microcanonical ensemble will correspond to the (long) timeaveraged statistics of the equations of motion (2.1). This hypothesis will be tested next using DNS of (2.1). In particular marginal pdfs $p_{\varepsilon}\left(\omega_{j}\right)$ for the scaled vorticity projections $\left\{\Omega_{j}\right\}$ can be compared directly with statistics from the DNS. Here we will focus on $p_{\varepsilon}\left(\omega_{1}\right)$, given by (3.15) and plotted in Fig. 5 , as well as the joint pdf $p_{\varepsilon}\left(\omega_{1}, \omega_{2}\right)$ contoured in Fig. 6 .

DNS of equation (2.1) is performed using the adaptive algorithm described in Ashbee et al. (2013). The majority of the runs to be discussed have been documented previously in EAM13. Briefly, equation (2.1) is solved for a uniform neutral gas with $N=100$, in domains $\mathrm{A}$ and $\mathrm{B}$, at a range of energies $(\varepsilon=-0.15,-0.1,-0.05,0,0.05,0.1,0.15)$ spanning the central region of the density of states function shown in Fig. 1. For each energy level and both domains, eight runs of $1000 \Gamma_{0}^{-1}$ time units are executed, during which the evolving vorticity projections $\Omega_{j}(t)$ are calculated and recorded at regular time intervals $\left(10 \Gamma_{0}^{-1}\right)$. Long time-averaged pdfs are then compiled from the histograms of the recorded data.

The red curves in Fig. 5 show the DNS results for $p_{\varepsilon}\left(\left|\omega_{1}\right|\right)$, which can be compared with the theoretical prediction (3.15) (blue curves). Notice that $p_{\varepsilon}\left(\left|\omega_{1}\right|\right)$ is plotted rather than $p_{\varepsilon}\left(\omega_{1}\right)$. Here we are exploiting the fact that $p_{\varepsilon}\left(\omega_{1}\right)$ is an even function to aid comparison with the DNS. This is necessary because in the high energy DNS (e.g. $\varepsilon=$ 0.1 ), particularly in domain $\mathrm{A}$, the system tends to become trapped for the duration of each run in a state with $\Omega_{1}$ either strictly positive or negative, producing asymmetric statistics. Good agreement is seen in Fig. 5, with much of the remaining discrepancy 

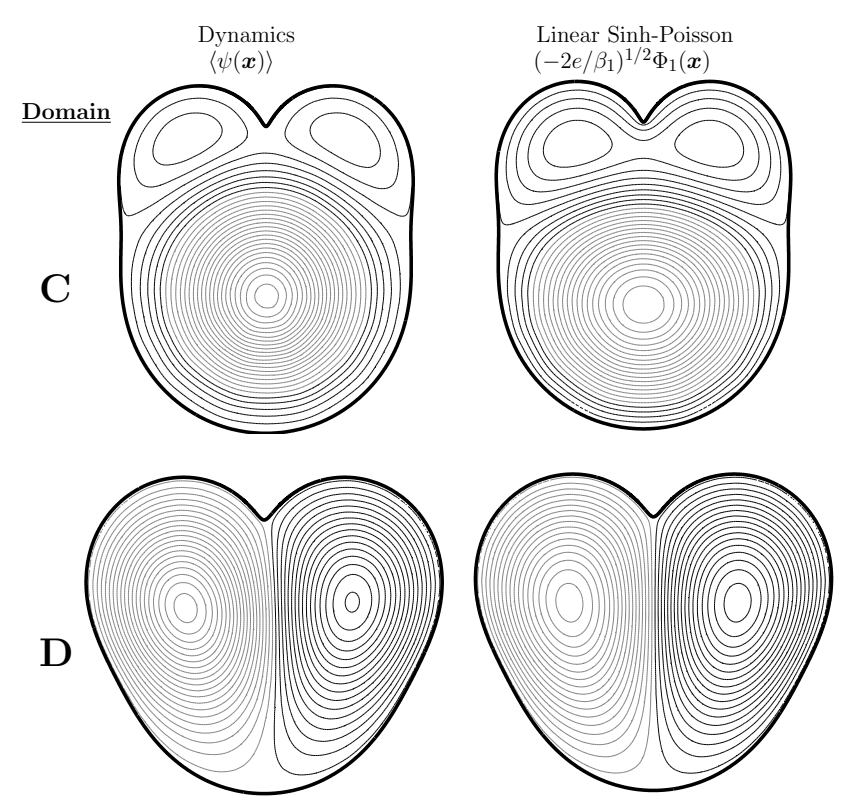

Figure 8. Left panels: Time-mean streamfunction (scaled by $N \Gamma_{0}$ as in $\left.\S 3.5\right)\langle\psi(\boldsymbol{x})\rangle$, calculated for time interval $1000 \Gamma_{0}^{-1}<t<3000 \Gamma_{0}^{-1}$, for dynamical runs with $N=100$ vortices in domains $\mathrm{C}$ and $\mathrm{D}$. The energies are $(\mathrm{C}) e=E / N^{2} \Gamma_{0}^{2}=0.013(\varepsilon=1.3)$ and (D) $e=0.011$ $(\varepsilon=1.1)$. Right panels: Linearised sinh-Poisson solution $\left(-2 e / \beta_{1}\right)^{1 / 2} \Phi_{1}(\boldsymbol{x})$ for the two domains. Contour intervals are the same in each panel.

arguably due to the finite integration length over which our statistics were compiled. In particular, the large contrast in the shape of $p_{\varepsilon}\left(\left|\omega_{1}\right|\right)$ between the two domains is equally apparent in the DNS as it is in the theory. Similarly, the DNS results in Fig. 6 (lower panels) agree well with the theoretical prediction of (3.16) (upper panels). The contrast between the two domains is again equally apparent in the DNS.

A further testable prediction is that the time-mean streamfunction $\langle\psi\rangle$ in the DNS, at energies in the matching region (low $e$, high $\varepsilon$ ), is at leading order given by (3.28). In particular the spatial structure of the condensate should match that of the first non-constant eigenfunction $\Phi_{1}(\boldsymbol{x})$. Domains $\mathrm{C}$ and D have been selected to provide an exacting numerical test that the correct spatial structure of $\langle\psi\rangle$ emerges, because as shown in Fig. 2, the two geometrically similar domains have very different first eigenfunctions. In $C, \Phi_{1}(\boldsymbol{x})$ has a tripole structure dominated by a single large vortex in the centre of the domain, whereas in $\mathrm{D} \Phi_{1}(\boldsymbol{x})$ has a left-right dipole structure.

The numerical runs for domains $\mathrm{C}$ and $\mathrm{D}$ are initialised with $N=100$ randomly placed vortices, and are executed just as described above for $\mathrm{A}$ and $\mathrm{B}$, but for a longer duration $\left(3000 \Gamma_{0}^{-1}\right.$ time units) and at higher energy $(\varepsilon=1.3$ for $\mathrm{C}$ and $\varepsilon=1.1$ for $\mathrm{D})$. High energy is necessary to prevent switches in the polarity of the circulation, as described 
above, occurring during the DNS. During each run, the exact instantaneous streamfunction at every point on a spatial grid is recorded. Note that, although the instantaneous streamfunction has logarithmic singularities at the locations of the vortices, provided that a vortex location is never exactly coincident with a grid-point the time-average at the grid-point remains well-defined. Moreover, due to the gentle nature of the logarithmic singularity, 'near-misses' where vortices pass close to grid-points do not dominate the time-average.

Fig. 8 shows a comparison between the time-mean streamfunction $\langle\psi\rangle$ calculated from the DNS (left panels) and the theoretical predictions (right panels). The DNS results capture the contrasting structures between the two domains. The $N=100$ vortex runs have been repeated with many sets of randomly generated initial conditions across a range of similar energies, and the emergence of the distinct structures in $\langle\psi\rangle$ in each domain has been found to be very robust. A detailed numerical investigation of the 'geometry-controlled transition' (see also Chavanis \& Sommeria 1996; Taylor et al. 2009) between the two structures, as the domain shape is varied smoothly between $\mathrm{C}$ and D is presented in Ashbee (2014).

\subsection{Phase-space sampling using a multi-canonical Markov chain Monte-Carlo (MC3)} method

The results of $\S 3$ can also be validated against statistics generated from repeatedly sampling the uniform distribution (see e.g. Campbell \& O'Neil 1991; Bühler 2002; Billam et al. 2014, EAM13). In the naive approach, the $N$ vortices are randomly distributed in the domain with uniform measure, and quantities of interest such as the energy and the vorticity projections $\Omega_{j}$ are calculated for the distribution in question and recorded. Repeating the process allows histograms to be generated from the recorded data, which then serve as estimates of the probability densities $p_{0}(\cdot)$ under the uniform ensemble. Probability densities under the microcanonical ensemble $p_{\varepsilon}(\cdot)$ can be generated from subsets of the data for which the energy lies in a shell centred on the energy $\varepsilon$ of interest.

If the point vortex system is ergodic, pdfs obtained by sampling the microcanonical canonical ensemble and pdfs obtained from long-time integrations of the dynamics (2.1), should be identical to within statistical error. For our calculations with $N=100$ this is indeed what we have found, i.e. there is no evidence of non-ergodicity, in contrast to the $N=6$ doubly-periodic calculations reported by Weiss \& McWilliams (1991).

The main drawback with the naive sampling method described above is that it is very expensive to generate data outside the central region of the density of states function illustrated in Fig. 1 (i.e. outside $-0.2 \lesssim \varepsilon \lesssim 0.3$ ), simply because the vast majority of random distributions of vortices will have energy within that range. To bypass the need to sample all energy levels simultaneously, and as a method of investigating energy levels outside of the central region, methods of sampling constant energy surfaces have been 
developed (Creutz 1983). Creutz's method has been used with apparent success in the point vortex context by Smith (1989) but has the disadvantages that (i) no proof exists that the microcanonical ensemble is accurately represented, and (ii) it does not allow direct calculation of the inverse temperature $\beta$ for comparison with theory.

An alternative method is multi-canonical Markov chain Monte-Carlo sampling (Berg 2000; Driscoll \& Maki 2007, MC3 hereafter). In its simplest form MC3 can be regarded as a method for sampling the tail regions of one-dimensional pdfs, and in our set-up the pdf in question is the density of states function $W_{\Gamma}(E)$. Our implementation of the MC3 algorithm closely follows the structure of the MATLAB code published by Driscoll \& Maki (2007, see discussion surrounding their Fig. 4) used therein to estimate the (known) binomial pdf of a one-dimensional random walk. For our problem, a Markov chain is initialised with a random distribution of vortices, and is updated at each step by moving a single vortex chosen at random, to a new random position in $\mathcal{D}$. The algorithm proceeds, for states in the positive tail region of $W_{\Gamma}(E)$, by accepting an update if the new distribution of vortices has greater energy $E$, and rejecting it with a certain probability if the updated $E$ is lower. The MC3 algorithm then uses the accumulated statistics of how $E$ changes under updates to adjust the rejection probabilities. The result is that, after a sufficient number of iterations, $W_{\Gamma}(E)$ is sampled with optimal efficiency far into the tail regions with an unbiased method.

MC3 has been used to sample $W_{\Gamma}(E)$ in domains A and B, where the circulations $\boldsymbol{\Gamma}$ are those of a uniform neutral gas with $N=100$. A Markov chain length of $1.5 \times 10^{7}$ was used for each iteration, of which the first third is a 'burn-in' which is not used when compiling the statistics. Note that the fact that only one vortex is moved each time the chain is advanced, can be used to accelerate the calculation of the energy and vorticity projections, allowing for efficient calculation of long chains. Twenty iterations were found to be necessary for the statistics to converge on the energy interval $-0.2 \lesssim \varepsilon \lesssim 2.5$. The failure of MC3 to significantly extend the statistics in the negative energy tail is due to the double exponential decay of $W_{\Gamma}(E)$ as $E \rightarrow-\infty$ (see Fig. 1).

Numerical differentiation was then used to obtain the corresponding inverse temperatures $\beta_{\Gamma}(E)=W_{\Gamma}^{\prime}(E) / W_{\Gamma}(E)$ which are plotted in Fig. 3 (red curves, $N \Gamma_{0}^{2} \beta_{\Gamma}$ plotted as a function of $\varepsilon=E / N \Gamma_{0}^{2}$ ). The calculated inverse temperatures can be compared with the theoretical predictions $\beta(\varepsilon)$ (fluctuation theory, green curves) and $\beta_{h}(e)$ (mean-field theory, blue curves). The left panels cover the central region $(-0.2<\varepsilon<0.3)$, which could equally well have been sampled using the naive method, and show excellent agreement between the MC3 sampled $\beta_{\boldsymbol{\Gamma}}(E)$ and the fluctuation theory $\beta(\varepsilon)$. The right panels, by contrast, focus on the positive tail region $(0 \lesssim \varepsilon<2)$ and show inverse temperatures close to the critical value $\beta=\beta_{1}$ (dashed line). On this scale, the MC3 results show a remarkable transition between close agreement with the fluctuation theory at lower 

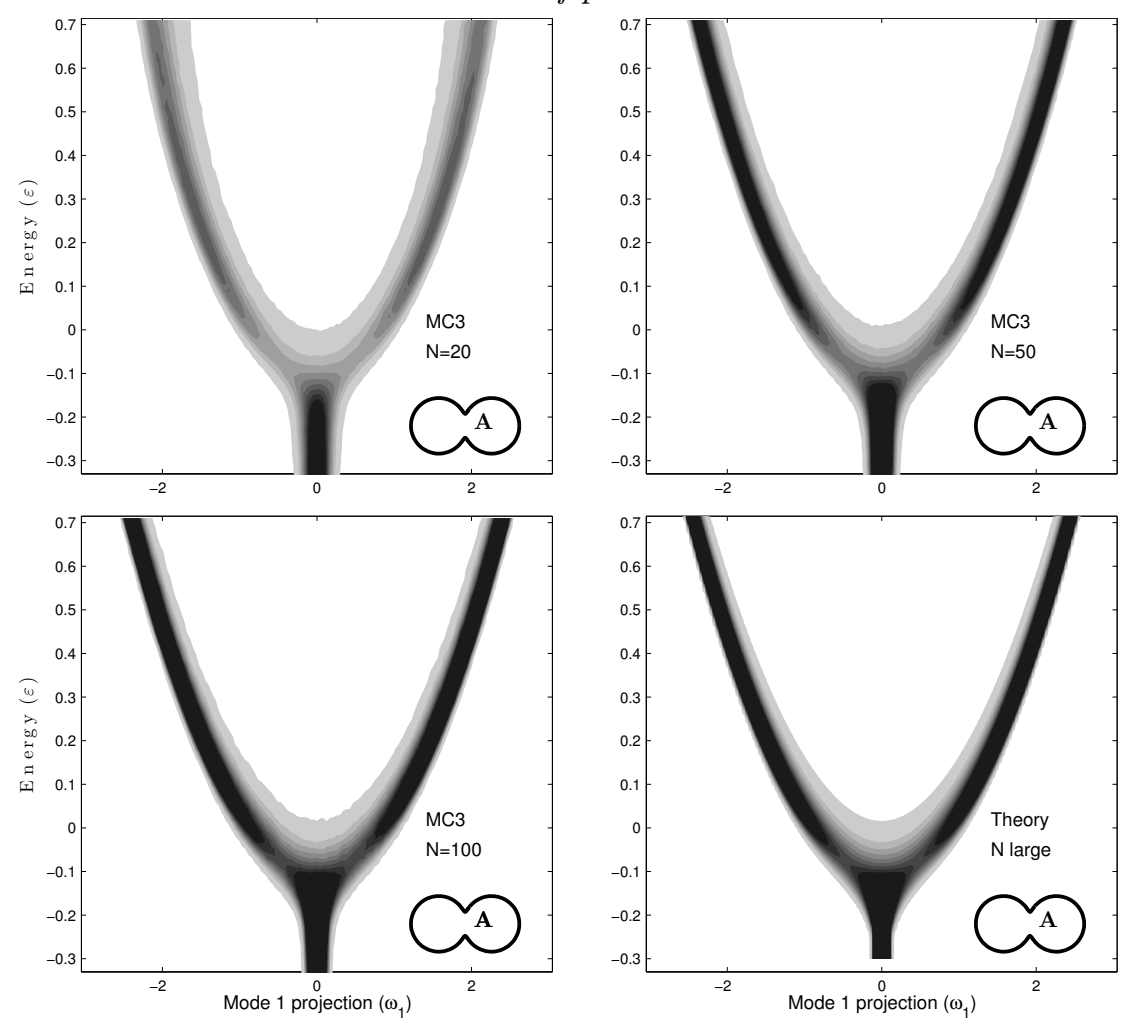

FiguRE 9. Top row: The microcanonical pdf $p_{\varepsilon}\left(\omega_{1}\right)$ of the vorticity projection $\Omega_{1}$ at energy $\varepsilon$, calculated for domain A with a uniform neutral gas, contoured as a function of $\left(\omega_{1}, \varepsilon\right)$. Top left: MC3 calculation with $N=20$ vortices. Top right: MC3 with $N=50$. Bottom left: MC3 with $N=100$. Bottom right: Theoretical prediction (eqn. 3.15).

energies, and close agreement with mean-field theory prediction $\beta_{h}(e)$ at higher energies. Accurate statistical validation of $\beta_{h}(e)$, which has not (to the authors' knowledge) been attempted previously, has therefore been made possible by MC3. The naive method is computationally too expensive by several orders of magnitude. To check the robustness of the result the MC3 calculation was repeated for domain A with $N=50$ vortices. Recall that, because Fig. 3 is plotted as a function of the thermodynamic energy $\varepsilon=E / N \Gamma_{0}^{2}$ rather than the hydrodynamic energy $e=\varepsilon / N$, the mean-field theory prediction $\beta_{h}(e)$ must be replotted on Fig. 3 when $N$ is changed. Nevertheless, the MC3 calculation (dark red curve) reveals that a clear transition between agreement with $\beta(\varepsilon)$ to agreement with $\beta_{h}(e)$ again takes place, i.e. both theories are correct within their range of validity.

Statistics compiled using MC3 can also be used as a check on the pdfs of the vorticity projections. In the lower panels of Fig. $4 p_{\varepsilon}\left(\omega_{1}\right)$, as calculated from histograms compiled from the MC3 data with $N=100$ vortices, is contoured. Excellent agreement with the theoretical predictions of (3.15) is evident in all three domains (A,C,D). It is clear from 
Fig. 4 that $N=100$ vortices are sufficient for the theory to be accurate, hence an obvious question relates to how the theory performs as $N$ is decreased. Fig. 9 compares $p_{\varepsilon}\left(\omega_{1}\right)$ estimated from MC3 calculations in domain A with $N=20,50$ and 100 vortices with the theoretical prediction. Even with as few as $N=20$ vortices, the phase transition between the uncondensed and condensed state is seen to occur at an almost identical energy $\varepsilon$. At higher energies there is a quantitative difference with the expected values of $\left|\Omega_{1}\right|$ somewhat lower for $N=20$ compared with the theory. An explanation for the difference is that $\varepsilon=0.7$ in Fig. 9 corresponds to a significantly higher value of the hydrodynamic energy $E$ at low $N(E=0.035$ for $N=20$ compared to $E=0.007$ for $N=100$ ). The former value of $E$ is sufficiently high for the sinh-Poisson equation (3.26) to predict significant nonlinearity in the $\langle\omega\rangle-\langle\psi\rangle$ relationship, and an associated change in the spatial structure of $\langle\psi\rangle$, causing it to differ significantly from that of $\Phi_{1}(\boldsymbol{x})$.

\section{Discussion and Conclusions}

A new methodology has been introduced above that, using the central limit theorem, allows pdfs from the microcanonical ensemble to be calculated and compared to timeaverages from DNS. Good agreement has been found. The pdfs in question are primarily those of the projections of the vorticity field onto a particular orthonormal basis, which has been argued to give a natural description of the modes of variability of the system (see also Chavanis \& Sommeria 1996). It is perhaps not surprising that the microcanonical ensemble, which is defined by conservation of energy, provides information most succinctly about the distribution of energy between modes that are orthogonal under the energy norm. In particular an exact expression (3.18) has been found which allows the complete energy spectrum to be calculated.

A natural topic for future investigation is to test the relevance of this predicted energy spectrum to simulations of two-dimensional Navier-Stokes and superfluid turbulence, particularly in the case where the area fraction covered by the vortices is very small, when the theory might be expected to be most relevant. In the case of superfluids, significant steps in this direction have been made by Billam et al. (2014), who have compared incompressible energy spectra computed from DNS of the Gross-Pitaevskii equations with energy spectra from point vortex integrations. Excellent agreement was found at low wavenumbers at a range of point vortex energies, showing that key aspects of the physics are well-captured by the point vortex model. The current work allows the energy spectrum in flows of this type to be calculated analytically, including in domains with geometry matching those of the experiments, and therefore promises detailed and flexible theoretical predictions of value to experimentalists.

A key question concerns the extent to which the point vortex approach remains relevant when non-equilibrium processes, such as vortex merger and filament formation in classi- 
cal turbulence or vortex annilihation in quantum turbulence, are active. Non-equilibrium processes act to change the vortex population, in classical turbulence by evolving both the distribution of vortex sizes and their number (empirical models of this process have been developed by Benzi et al. 1992; Dritschel et al. 2008), and in quantum turbulence by reducing the number of vortices. Our (testable) hypothesis is that a point vortex description will be successful whenever $\tau_{e} \ll \tau_{n e}$, where $\tau_{e}$ is the characteristic time-scale for the point vortex system to relax to equilibrium (i.e. forget initial conditions), and $\tau_{n e}$ is the time-scale over which non-equilibrium processes act to change the vortex population. If $\tau_{e} \ll \tau_{n e}$ the equilibrium statistics of the point vortex model will remain relevant to those of the evolving turbulent flow, because the turbulent flow in this case adjusts sufficiently rapidly that no memory of its changing vortex population is retained. The good correspondence between the point vortex model and the Gross-Pitaevskii calculations of Billam et al. (2014) suggests that, at least in some circumstances, $\tau_{e} \ll \tau_{n e}$ in typical numerical simulations of 2D quantum turbulence. Similarly, a preliminary study of DNS of decaying 2D classical turbulence by the first author and collaborators suggests that, at least at late times in typical simulations, point vortex predictions correspond closely to the observed energy spectrum.

An important point concerning the use of the point vortex model to interpret DNS of classical or quantum turbulence is that, because the vortex population evolves in the turbulent flow, the mapping from the point vortex model to the turbulent flow also evolves in time. In particular the point vortex energy $\varepsilon$ corresponding to the turbulent flow will change as the vortex population evolves, even as the fluid energy $E_{F}$ of the turbulent flow is approximately conserved. Under the empirical model of vortex population evolution in classical turbulence suggested by Dritschel et al. (2008), $\varepsilon$ can be shown to increase with time, consistent with the $2 \mathrm{D}$ turbulent flow evolving towards a regime favouring like-signed vortex clustering and eventual Onsager-Kraichnan condensation. In this picture, which will be explored in more detail in future work, the combination of the point vortex model and any suitable model of vortex population evolution provides a route to a quantitative statistical understanding of upscale energy transfer in 2D classical turbulence.

The main focus of much of this work has been a detailed investigation of OnsagerKraichnan condensation in the point vortex model, addressing the question of under what circumstances a steady time-mean flow spontaneously emerges in a given set-up. It is clear from both the theoretical results and those from statistical sampling of phase-space that condensation is a spontaneous symmetry-breaking in the system. The pitchfork-like bifurcations shown in Fig. 4 resemble those seen in second-order phase transitions in (for example) the Ising model of ferromagnetism, with the first vorticity projection $\Omega_{1}$ taking the role of a global order parameter. However, there are obstacles to developing 
this analogy because the point vortex system is a long-range interacting Hamiltonian system, and is resistant to many of the standard methods of statistical mechanics (e.g. Campa et al. 2009). An interesting new result is that, at the onset of condensation, the energy spectrum becomes saturated, with any additional energy going exclusively into the condensate mode. The saturated spectrum can be found explicitly (see eqn. 3.22).

Many of the remaining results relate to how condensation is influenced by the domain shape. The geometry of the domain determines the eigenvalues $\left\{\beta_{j}\right\}$, and in turn these control, for example, the amplitude of fluctuations of the energy in the condensate mode, and the scaling of the transition probability $p_{\varepsilon}\left(\omega_{1}=0\right)$, which controls the frequency of switches in the polarity of the condensate mean flow. The behaviour is most sensitive to the size of the spectral gap $\beta_{1}-\beta_{2}$. It is important to emphasise that, because the point vortex system is a long-range interacting one, there is no limit in which the shape of the domain does not influence the statistics of the flow. This point is particularly relevant to the study of two-dimensional turbulence in doubly-periodic domains. Rather than somehow representing the flow on an infinite domain, as might be supposed, the specific nature of the doubly-periodic geometry influence the statistics just as profoundly as that of the bounded domains studied here.

JGE would like to acknowledge the hospitality of the Kavli Institute for Theoretical Physics, where this research was supported in part by the National Science Foundation under Grant No. NSF PHY11-25915. The authors are grateful to J. Vanneste for suggesting the MC3 sampling method.

\section{Appendix A. Some mathematical details pertaining to section 3}

\section{A.1. The Hamiltonian (3.11) in the limit $N \rightarrow \infty$}

Here we aim to justify carefully the approximation of (3.11) by (3.12) in the limit $N \rightarrow \infty$. We will establish the result by first considering an application of the multivariate CLT to a $3 M$-component random vector $\boldsymbol{Z}_{i_{k}}^{k}$ to obtain the distribution of its sample mean $\boldsymbol{S}^{k}$ in the limit $N \rightarrow \infty$. Here $M$ denotes the number of eigenfunctions at which expansions such as (3.2) are truncated. The random vectors $\left\{\boldsymbol{Z}_{i_{k}}^{k}\right\}$, are associated with the contributions 
of those vortices, with indices $i_{k}$, that have circulation $\bar{\Gamma}_{k}$. They are given by

$$
\boldsymbol{Z}_{i_{k}}^{k}=\left(\begin{array}{c}
\Phi_{1}\left(\boldsymbol{X}_{i_{k}}\right) \\
\vdots \\
\Phi_{M}\left(\boldsymbol{X}_{i_{k}}\right) \\
N^{-1 / 2} \bar{\Gamma}_{k} \Gamma_{0}^{-1}\left(\Phi_{1}\left(\boldsymbol{X}_{i_{k}}\right)^{2}-1\right) \\
\vdots \\
N^{-1 / 2} \bar{\Gamma}_{k} \Gamma_{0}^{-1}\left(\Phi_{M}\left(\boldsymbol{X}_{i_{k}}\right)^{2}-1\right) \\
N^{-1 / 2} \bar{\Gamma}_{k} \Gamma_{0}^{-1} \Phi_{1}\left(\boldsymbol{X}_{i_{k}}\right) \\
\vdots \\
N^{-1 / 2} \bar{\Gamma}_{k} \Gamma_{0}^{-1} \Phi_{M}\left(\boldsymbol{X}_{i_{k}}\right)
\end{array}\right) \text { and } \boldsymbol{S}^{k}=\frac{1}{\left(\alpha_{k} N\right)^{1 / 2}} \sum_{i_{k}=1}^{\alpha_{k} N} \boldsymbol{Z}_{i_{k}}^{k}=\left(\begin{array}{c}
\Omega_{1}^{k} \\
\vdots \\
\Omega_{M}^{k} \\
T_{1}^{k} \\
\vdots \\
T_{M}^{k} \\
S_{1}^{k} \\
\vdots \\
S_{M}^{k}
\end{array}\right) .
$$

The $\left\{\boldsymbol{Z}_{i_{k}}^{k}\right\}$ have been designed in order that their normalised sums $\boldsymbol{S}^{k}$ have components $\left\{\Omega_{j}^{k}, T_{j}^{k}, S_{j}^{k}\right\}$, where $\Omega_{j}^{k}$ has been previously defined in (3.3), whereas $T_{j}^{k}$ and $S_{j}^{k}$ are defined by the sums in (A 1). These random variables have zero mean, and by design satisfy

$$
\sum_{k=1}^{K} \alpha_{k}^{1 / 2} \frac{\bar{\Gamma}_{k}}{\Gamma_{0}} \Omega_{j}^{k}=\Omega_{j}, \quad \sum_{k=1}^{K} \alpha_{k}^{1 / 2} \frac{\bar{\Gamma}_{k}}{\Gamma_{0}} T_{j}^{k}=T_{j}, \quad \sum_{k=1}^{K} \alpha_{k}^{1 / 2} \frac{\bar{\Gamma}_{k}}{\Gamma_{0}} S_{j}^{k}=S_{j},
$$

where the random variables $\left\{\Omega_{j}, T_{j}, S_{j}\right\}$ are those appearing in connection with (3.11) (recall that $T_{j}=R_{j j}-1$ ).

The multivariate CLT must be used because the components of $\boldsymbol{Z}_{i_{k}}^{k}$ are not independent. The multivariate CLT states that, provided that all components of the covariance matrix exist, in the limit $N \rightarrow \infty$ the random vector $\boldsymbol{S}^{k}$ will be distributed as

$$
\boldsymbol{S}^{k} \sim \mathcal{N}(\mathbf{0}, \mathbf{\Sigma})
$$

where $\boldsymbol{\Sigma}=\mathbb{E}\left(\boldsymbol{Z}_{i_{k}}^{k} \boldsymbol{Z}_{i_{k}}^{k}\right)$ is the covariance matrix associated with $\boldsymbol{Z}_{i_{k}}^{k}$. It is straightforward to calculate the entries in the covariance matrix $\boldsymbol{\Sigma}$ (note that, because covariance matrices are symmetric, only entries with $p \leqslant q$ need be given explicitly)

$$
(\boldsymbol{\Sigma})_{p q}= \begin{cases}\delta_{p^{\prime} q^{\prime}} & 1 \leqslant p, q \leqslant M \\ N^{-1 / 2} \bar{\Gamma}_{k} \Gamma_{0}^{-1}\left[\Phi_{p^{\prime}} \Phi_{q^{\prime}}^{2}\right] & 1 \leqslant p \leqslant M, M+1 \leqslant q \leqslant 2 M \\ N^{-1 / 2} \bar{\Gamma}_{k} \Gamma_{0}^{-1} \delta_{p^{\prime} q^{\prime}} & 1 \leqslant p \leqslant M, 2 M+1 \leqslant q \leqslant 3 M \\ N^{-1} \bar{\Gamma}_{k}^{2} \Gamma_{0}^{-2}\left(\left[\Phi_{p^{\prime}}^{2} \Phi_{q^{\prime}}^{2}\right]-1\right) & M+1 \leqslant p, q \leqslant 2 M \\ N^{-1} \bar{\Gamma}_{k}^{2} \Gamma_{0}^{-2}\left[\Phi_{p^{\prime}}^{2} \Phi_{q^{\prime}}\right] & M+1 \leqslant p \leqslant 2 M, 2 M+1 \leqslant q \leqslant 3 M \\ N^{-1} \bar{\Gamma}_{k}^{2} \Gamma_{0}^{-2} \delta_{p^{\prime} q^{\prime}} & 2 M+1 \leqslant p, q \leqslant 3 M\end{cases}
$$

Here square brackets [.] denote the area average over $\mathcal{D}, \delta_{p q}$ is the Kronecker delta, and primes on the indices denote that the modulus with respect to $M$ is intended (i.e. $p^{\prime}=p$ mod $M$ ). The important point to take from (A 4) is simply that all covariances are finite, justifying the use of the CLT. Further, in the limit $N \rightarrow \infty$ the matrix is dominated by 
the diagonal entries with $1 \leqslant p=q \leqslant M$, hence in the limit $N \rightarrow \infty$ we can neglect all components in $\mathbf{S}^{k}$ except the $\left\{\Omega_{j}^{k}\right\}$ which are iid with $\Omega_{j}^{k} \sim \mathcal{N}(0,1)$. Summing the Gaussian distributed random variables in (A 2), and then taking the limit $M \rightarrow \infty$, the result that the vorticity projections are iid with $\Omega_{j} \sim \mathcal{N}(0,1)$ is recovered, along with the fact that at leading order in $N$ the variables $\left\{T_{j}\right\}$ and $\left\{S_{j}\right\}$ can be neglected, as required for (3.12).

\section{A.2. Derivation of the energy spectrum}

Here, the details are given for the result (3.18), which expresses the microcanonical average of $\left\langle\Omega_{j}^{2}\right\rangle$ in terms of the density of states function $W(\varepsilon)$ and its derivatives. First note that

$$
\left\langle\Omega_{j}^{2}\right\rangle=\int_{-\infty}^{\infty} \omega_{j}^{2} p_{\varepsilon}\left(\omega_{j}\right) \mathrm{d} \omega_{j},
$$

where $p_{\varepsilon}\left(\omega_{j}\right)$ is the microcanonical pdf of $\Omega_{j}$ given by (3.15). Next, note that

$$
\begin{aligned}
& \int_{-\infty}^{\infty} \omega_{j}^{2} p_{\varepsilon}\left(\omega_{j}\right) \mathrm{d} \omega_{j}=\frac{1}{F^{\boldsymbol{\beta}}\left(\varepsilon-\varepsilon_{0}\right)} \int_{-\infty}^{\infty} F^{\boldsymbol{\beta}(j)}\left(\varepsilon-\varepsilon_{0}+\frac{\omega_{j}^{2}-1}{2 \beta_{j}}\right) \frac{\omega_{j}^{2}}{\sqrt{2 \pi}} \exp \left(-\frac{\omega_{j}^{2}}{2}\right) \mathrm{d} \omega_{j} \\
& =\frac{1}{W(\varepsilon)} \int_{-\infty}^{\infty} \frac{A^{\boldsymbol{\beta}(j)}(k)}{(2 \pi)^{3 / 2}} \exp \left(\mathrm{i}\left(\varepsilon-\varepsilon_{0}\right) k+\mathrm{i} \phi^{\boldsymbol{\beta}(j)}(k)-\frac{\mathrm{i} k}{2 \beta_{j}}\right)\left(\int_{-\infty}^{\infty} \omega_{j}^{2} \exp \left(\frac{\mathrm{i} k \omega_{j}^{2}}{2 \beta_{j}}-\frac{\omega_{j}^{2}}{2}\right) \mathrm{d} \omega_{j}\right) \mathrm{d} k \\
& =\frac{1}{W(\varepsilon)} \int_{-\infty}^{\infty} \frac{A^{\boldsymbol{\beta}(j)}(k)}{(2 \pi)} \frac{\left(\beta_{j}^{2}+\mathrm{i} k \beta_{j}\right) \exp \left(\mathrm{i}\left(\varepsilon-\varepsilon_{0}\right) k+\mathrm{i} \phi^{\boldsymbol{\beta}(j)}(k)-\mathrm{i} k /\left(2 \beta_{j}\right)+\frac{1}{2} \mathrm{itan}^{-1}\left(k / \beta_{j}\right)\right)}{\left(1+k^{2} / \beta_{j}^{2}\right)^{1 / 4}\left(k^{2}+\beta_{j}^{2}\right)} \mathrm{d} k \\
& =\frac{1}{W(\varepsilon)} \int_{-\infty}^{\infty} \frac{A^{\boldsymbol{\beta}}(k)\left(\beta_{j}^{2}+\mathrm{i} k \beta_{j}\right)}{2 \pi\left(k^{2}+\beta_{j}^{2}\right)} \exp \left(\mathrm{i}\left(\varepsilon-\varepsilon_{0}\right) k+\mathrm{i} \phi^{\boldsymbol{\beta}}(k)\right) \mathrm{d} k .
\end{aligned}
$$

A result equivalent to (A 6) was obtained by EAM13 (see their eqn. 45, and note that $\left.a_{j j}+1=\left\langle\Omega_{j}^{2}\right\rangle\right)$.

To obtain (3.18) it remains to calculate

$$
\begin{aligned}
\frac{\partial W}{\partial \beta_{j}}(\varepsilon) & =\frac{1}{2 \pi} \int_{-\infty}^{\infty}\left(\frac{\partial A^{\boldsymbol{\beta}}}{\partial \beta_{j}}+\mathrm{i} A^{\boldsymbol{\beta}} \frac{\partial \phi^{\boldsymbol{\beta}}}{\partial \beta_{j}}\right) \exp \left(\mathrm{i}\left(\varepsilon-\varepsilon_{0}\right) k+\mathrm{i} \phi^{\boldsymbol{\beta}}(k)\right) \mathrm{d} k \\
& =\frac{1}{2 \pi} \int_{-\infty}^{\infty} \frac{k^{2}\left(\beta_{j}+\mathrm{i} k\right)}{2 \beta_{j}^{2}\left(k^{2}+\beta_{j}^{2}\right)} A^{\boldsymbol{\beta}}(k) \exp \left(\mathrm{i}\left(\varepsilon-\varepsilon_{0}\right) k+\mathrm{i} \phi^{\boldsymbol{\beta}}(k)\right) \mathrm{d} k \\
& =\frac{1}{2 \pi} \int_{-\infty}^{\infty} \frac{\left(\beta_{j}+\mathrm{i} k\right)}{2}\left(\frac{1}{\beta_{j}^{2}}-\frac{1}{\left(k^{2}+\beta_{j}^{2}\right)}\right) A^{\boldsymbol{\beta}}(k) \exp \left(\mathrm{i}\left(\varepsilon-\varepsilon_{0}\right) k+\mathrm{i} \phi^{\boldsymbol{\beta}}(k)\right) \mathrm{d} k \\
& =\frac{1}{2 \beta_{j}}\left(W(\varepsilon)+W^{\prime}(\varepsilon) / \beta_{j}-\left\langle\Omega_{j}^{2}\right\rangle W(\varepsilon)\right),
\end{aligned}
$$

from which (3.18) follows.

\section{A.3. High energy asymptotics of (3.13)}

Here the high energy $(\varepsilon \rightarrow \infty)$ asymptotics of the density of states function $W(\varepsilon)$ is considered. We have not managed to find a direct method to approximate (3.13) as 


\begin{tabular}{c|ccc}
\hline \multicolumn{1}{c}{$k$} & $B_{k}$ & $A_{j k} \quad(j \geqslant 2)$ & $A_{1 k}$ \\
$k=1$ & $\beta_{1}$ & $\frac{\beta_{j}}{\beta_{j}-\beta_{1}}$ & $1-s_{1}$ \\
$k=2$ & $-\frac{1}{2}$ & $-\frac{\beta_{j}}{2\left(\beta_{j}-\beta_{1}\right)^{2}}$, & $-\frac{s_{2}}{2 \beta_{1}}$ \\
$k=3$ & $\frac{1}{4 \beta_{1}}\left(s_{1}-1\right)$ & $\frac{1}{4}\left(\frac{3 \beta_{j}}{\left(\beta_{j}-\beta_{1}\right)^{3}}+\frac{\left(s_{1}-1\right) \beta_{j}}{\beta_{1}\left(\beta_{j}-\beta_{1}\right)^{2}}\right)$ & $-\frac{1}{4 \beta_{1}^{2}}\left(3 s_{3}+s_{2}\left(s_{1}-1\right)\right)$ \\
$-\frac{1}{8 \beta_{1}^{2}}\left(\left(s_{1}-1\right)^{2}+3 s_{2}\right)$ & etc. & etc.
\end{tabular}

TABLE 1. Coefficients in the expansion (A 9) of (A 7-A 8). Here the constants $\left\{s_{1}, s_{2}, s_{3}\right\}$ refer to summations $s_{1}=\sum_{j=2}^{\infty} \beta_{1}^{2} / \beta_{j}\left(\beta_{j}-\beta_{1}\right), s_{2}=\sum_{j=2}^{\infty} \beta_{1}^{2} /\left(\beta_{j}-\beta_{1}\right)^{2}, s_{3}=\sum_{j=2}^{\infty} \beta_{1}^{3} /\left(\beta_{j}-\beta_{1}\right)^{3}$.

$\varepsilon \rightarrow \infty$. The following indirect method is used instead. Consider the equations

$$
\begin{aligned}
\varepsilon-\varepsilon_{0} & =-\frac{1}{2} \sum_{j=1}^{\infty} \frac{\left\langle\Omega_{j}^{2}\right\rangle-1}{\beta_{j}}, \\
\left(\partial_{\varepsilon}+\beta(\varepsilon)\right)\left\langle\Omega_{j}^{2}\right\rangle & =\beta_{j}\left(\left\langle\Omega_{j}^{2}\right\rangle-1\right) \quad(j \geqslant 1) .
\end{aligned}
$$

Here (A 7) is the microcanonical ensemble average of (3.12), and (A 8) follows from differentiating of (A 6) with respect to $\varepsilon$. Eqns. (A 7 -A 8) can also be obtained by the cumulant expansion method (c.f. equations 37 and 39 of EAM13).

To study (A 7-A 8) asymptotically in the limit $\varepsilon \rightarrow \infty$, it is necessary to take as a starting point the fact that all of the energy at leading order is contained in the $j=1$ mode, i.e. that associated with condensation. A solution based on the following expansion in the small parameter $\left(\varepsilon-\varepsilon_{0}\right)^{-1}$, can then be sought

$$
\begin{aligned}
& \left\langle\Omega_{1}^{2}\right\rangle=-2 \beta_{1}\left(\varepsilon-\varepsilon_{0}\right)+\sum_{k=0}^{\infty} A_{1 k}\left(\varepsilon-\varepsilon_{0}\right)^{-k}, \\
& \left\langle\Omega_{j}^{2}\right\rangle=\sum_{k=0}^{\infty} A_{j k}\left(\varepsilon-\varepsilon_{0}\right)^{-k}, \quad(j \geqslant 2) \\
& \beta(\varepsilon)=\sum_{k=0}^{\infty} B_{k}\left(\varepsilon-\varepsilon_{0}\right)^{-k} .
\end{aligned}
$$

The values obtained when the expansion (A 9) is inserted into (A 7-A 8), and powers in $\left(\varepsilon-\varepsilon_{0}\right)^{-1}$ are equated are shown in Table 1 . The expansion for $\beta(\varepsilon)$ can be integrated to give

$$
W(\varepsilon) \approx \frac{W_{0}}{\left(\varepsilon-\varepsilon_{0}\right)^{1 / 2}} \exp \left(\beta_{1}\left(\varepsilon-\varepsilon_{0}\right)-\sum_{k=1}^{\infty} \frac{B_{k+1}}{k\left(\varepsilon-\varepsilon_{0}\right)^{k}}\right) .
$$

for $W_{0}$ constant. The $\left\{\beta_{j}\right\}$-dependency of the constant $W_{0}$ can be obtained by demanding 
consistency with (3.18), giving after some working,

$$
W_{0}=W_{00}\left(-\beta_{1}\right)^{1 / 2} \exp \left(-\frac{1}{2} \sum_{j=2}^{\infty} \log \left(1-\frac{\beta_{1}}{\beta_{j}}\right)+\frac{\beta_{1}}{\beta_{j}}\right) .
$$

where $W_{00}$ is a constant independent of the $\left\{\beta_{j}\right\}$. Finally, consistency with the single variable pdf (2.16) in the limit $\beta_{j} \rightarrow \infty(j \geqslant 2)$ requires $W_{00}=\pi^{-1 / 2}$, resulting in (3.20).

A notable feature in Table 1 is that the calculated coefficients $\left\{B_{k}\right\}$ in the expansion of $\beta(\varepsilon)$ are negative, provided that $s_{1}>1$, which appears to be the case in all domains. Under this assumption on $s_{1}$, it turns out to be possible to prove inductively that $B_{k}<0$ for all $k$. The proof by induction starts with the following set of three assumptions: that for some positive $k$, for all $0 \leqslant i \leqslant k-1$ firstly $A_{1 i}<0$, secondly $A_{j i}>0$ for all $j \geqslant 2$, and thirdly $B_{i+1}<0$. Inspection of table 1 reveals that these assumptions hold true for $k=1$ and $k=2$. Inserting the expansion (A 9) into equation (A 8) with $j=1$, and then equating terms of order $\left(\varepsilon-\varepsilon_{0}\right)^{-k}$ results in

$$
B_{k+1}=\frac{1}{2 \beta_{1}}\left(-\left(s_{1}-1\right) B_{k}+\sum_{i=1}^{k-2} A_{1 i} B_{k-i}-\left(k-\frac{1}{2}\right) A_{1, k-1}\right)<0, \quad(k \geqslant 2)
$$

because, under our assumptions above, every term inside the brackets is positive. Considering next $(\mathrm{A} 8)$ with $j \geqslant 2$, and again equating terms of order $\left(\varepsilon-\varepsilon_{0}\right)^{-k}$ we have

$$
A_{j k}=\frac{1}{\beta_{1}-\beta_{j}}\left(\left(k-\frac{1}{2}\right) A_{j, k-1}-\sum_{i=2}^{k} B_{i} A_{j, k-i}\right)>0, \quad(k \geqslant 1)
$$

again using the three assumptions. Finally, it follows from the expansion of (A 7) that

$$
A_{1 k}=-\beta_{1} \sum_{j=2}^{\infty} \frac{A_{j k}}{\beta_{j}}<0
$$

In summary, we have proved that if our three assumptions hold for some $k$, then they will also hold for $k+1$. Given that they hold for $k=2$, inductively they must hold for all $k$, i.e. $B_{k}<0$ for all $k$, establishing the result.

\section{REFERENCES}

Ashbee, T. L. 2014 Dynamics and statistical mechanics of point vortices in bounded domains. $\mathrm{PhD}$ thesis, University College London.

Ashbee, T. L., Esler, J. G. \& McDonald, N. R. 2013 Generalized Hamiltonian point vortex dynamics on arbitrary domains using the method of fundamental solutions. J. Comput. Phys. 246, 289-303.

BAusch, J. 2013 On the efficient calculation of a linear combination of chi-square random variables with an application in counting string vacua. J. Phys. A: Math. Theor. 46, 505202.

Benzi, R., Colella, M., Briscolini, M. \& Santangelo, P. 1992 A simple point vortex model for two-dimensional decaying turbulence. Phys. Fluids 4, 1036-1039. 
Berg, B. A. 2000 Introduction to multicanonical Monte Carlo simulations. Fields Inst. Commun. 26, 124.

Billam, T. P., Reeves, M. T., Anderson, B. P. \& Bradley, A. S. 2014 Onsager-Kraichnan condensation in decaying two-dimensional quantum turbulence. Phys. Rev. Lett. 112, 145301

Book, D. L., Fisher, S. \& McDonald, B. E. 1975 Steady-state distributions of interacting discrete vortices. Phys. Rev. Lett. 34, 4-8.

Bos, W. J. T., NeffaA, S. \& Schneider, K. 2008 Rapid generation of angular momentum in bounded magnetized plasma. Phys. Rev. Lett. 101, 235003.

Bradley, A. S. \& Anderson, B. P. 2012 Energy spectra of vortex distributions in twodimensional quantum turbulence. Phys. Rev. X 2, 041001.

BüHLER, O. 2002 Statistical mechanics of strong and weak point vortices in a cylinder. Phys. Fluids 14 (7), 2139-2149.

Campa, A., Dauxois, T. \& Ruffo, S. 2009 Statistical mechanics and dynamics of solvable models with long-range interactions. Phys. Rep. 480, 57-159.

Campbell, L. J. \& O’Neil, K. 1991 Statistics of two-dimensional point vortices and highenergy vortex states. J. Stat. Phys. 65, 495-529.

Chavanis, P.-H. \& Sommeria, J. 1996 Classification of self-organized vortices in twodimensional turbulence: The case of a bounded domain. J. Fluid Mech. 314, 267-297.

Chertkov, M., Connaughton, C., Kolokolov, I. \& Lebedev, V. 2007 Dynamics of energy condensation in two-dimensional turbulence. Phys. Rev. Lett. 99, 084501.

Clercx, H. J. H., MaAssen, S. R. \& van Heisst, G. J. F. 1998 Spontaneous spin-up during the decay of $2 \mathrm{~d}$ turbulence in a square container with rigid boundaries. Phys. Rev. Lett. 80, 5129-5132.

Clercx, H. J. H., Nielsen, A. H., Torres, D. J. \& Coutsias, E. A. 2001 Two-dimensional turbulence in square and circular domains with no-slip walls. Eur. J. Mech. B-Fluid 20 (4), $557-576$.

Creutz, M. 1983 Microcanonical Monte Carlo simulation. Phys. Rev. Lett. 50, 1411-1414.

Debnath, L. \& Mikusiński, P. 2005 Introduction to Hilbert spaces with applications, 3rd edn. Amsterdam: Elsevier.

Driscoll, T. A. \& MAKI, K. L. 2007 Searching for rare growth factors using multicanonical Monte Carlo methods. SIAM Rev. 49, $673-692$.

Dritschel, D. G., Lucia, M. \& Poje, A. C. 2015 Equilibrium statistics and dynamics of point vortex flows on the sphere. Phys. Rev. E 91, 063014.

Dritschel, D. G., Scott, R. K., Macaskill, C., Gottwald, G. A. \& Tran, C. V. 2008 Unifying scaling theory for vortex dynamics in two-dimensional turbulence. Phys. Rev. Lett. 101, 094501.

EdWARds, S. F. \& TAYLOR, J. B. 1974 Negative temperature states of two-dimensional plasmas and vortex fluids. Proc. R. Soc. A 336 (1606), 257-271.

Esler, J. G., Ashbee, T. L. \& McDonald, N. R. 2013 Statistical mechanics of a neutral point-vortex gas at low energy. Phys. Rev. E 88, 012109.

Eyink, G. L. \& Sreenivasan, K. R. 2006 Onsager and the theory of hydrodynamic turbulence. Rev. Mod. Phys. 78, 87-135. 
Flucher, M. \& Gustafsson, B. 1999 Vortex motion in two dimensional hydrodynamics. New York: Springer.

Joyce, G. \& Montgomery, D. 1973 Negative temperature states for a two-dimensional guiding center plasma. J. Plasma Phys. 10, 107-121.

Kiessling, M. K.-H. 1995 Negative temperature bounds for 2d vorticity compounds. Lett. Math. Phys. 34, 49-57.

Kunin, I. A., Hussain, F. \& Zhou, X. 1994 Dynamics of a pair of vortices in a rectangle. Int. J. Engng. Sci. 32 (12), 1835-1844.

LiN, C. C. 1941a On the motion of vortices in two dimensions-I. Existence of the KirchhoffRouth function. Proc. Natl Acad. Sci. 27, 570-575.

MCDonALD, B. E. 1974 Numerical calculation of nonunique solutions of a two-dimensional sinh-Poisson equation. J. Comput. Phys. 16 (4), 360 - 370, 0021-9991.

Miller, J. 1990 Statistical mechanics of the Euler equation in two dimensions. Phys. Rev. Lett. 65, 2137-2140.

Naso, A., Chavanis, P.-H. \& Dubrulle, B. 2010 Statistical mechanics of two-dimensional Euler flows and minimum enstrophy states. Eur. Phys. J. B $\mathbf{7 7}$ (2), 187-212.

Neely, T. W., Bradley, A. S., Samson, E. C., Rooney, S. J., Wright, E. M., LaW, K. J. H., Carretero-González, R., Kevrekidis, P. G., Davis, M. J. \& Anderson, B. P. 2013 Characteristics of two-dimensional quantum turbulence in a compressible superfluid. Phys. Rev. Lett. 111, 235301.

Newton, P. K. 2001 The N-Vortex Problem: Analytical Techniques. New York: Springer-Verlag.

Onsager, L. 1949 Statistical hydrodynamics. Il Nuovo Cimento (1943-1954) 6, 279-287.

PARet, J. \& TABeling, P. 1998 Intermittency in the two-dimensional inverse cascade of energy: Experimental observations. Phys. Fluids 10, 3126.

Pointin, Y. B. \& Lundgren, T. S. 1976 Statistical mechanics of two-dimensional vortices in a bounded container. Phys. Fluids 19 (10), 1459-1470.

Richardson, S. 1981 Some Hele-Shaw flows with time-dependent free boundaries. J. Fluid Mech. 102, 263-278.

Robert, R. 1991 A maximum entropy principle for two-dimensional Euler equations. J. Stat. Phys. 65, 531-553.

Shats, M. G., XiA, H. \& Punzmann, H. 2005 Spectral condensation of turbulence in plasmas and fluids and its role in low-to-high phase transitions in toroidal plasma. Phys. Rev. E 71, 046409 .

Simula, T., Davis, M. J. \& Helmerson, K. 2014 Emergence of order from turbulence in an isolated planar superfluid. Phys. Rev. Lett. 113, 165302.

Sмiтh, R. A. 1989 Phase transition behavior in a negative-temperature guiding-center plasma. Phys. Rev. Lett. 63, 1479-1482.

TAylor, J. B., Borchardt, M. \& Helander, P. 2009 Interacting vortices and spin-up in two-dimensional turbulence. Phys. Rev. Lett. 102, 124505.

Touchette, H. 2009 The large deviation approach to statistical mechanics. Phys. Rep. 478 (13), 1-69.

Trefethen, L. N. 2000 Spectral Methods in MATLAB. Philadelphia: SIAM.

Weiss, J. B. \& McWilliams, J. C. 1991 Nonergodicity of point vortices. Phys. Fluids A 3 (5), $835-844$ 
Xiao, Z., Wan, M., Chen, S. \& Eyink, G. L. 2009 Physical mechanism of the inverse energy cascade of two-dimensional turbulence: a numerical investigation. J. Fluid Mech. 619, 1-44. 